\title{
Membrane-containing icosahedral bacteriophage PRD1: The Dawn of Viral Lineages
}

\section{Oksanen, Hanna M.}

Springer

2019

Oksanen , H M \& Abrescia , N G A 2019 , Membrane-containing icosahedral bacteriophage PRD1 : The Dawn of Viral Lineages . in U F Greber (ed.), PHYSICAL VIROLOGY : VIRUS STRUCTURE AND MECHANICS . Advances in Experimental Medicine and Biology, vol. 1215 , Springer , pp. 85-109 . https://doi.org/10.1007/978-3-030-14741-9_5

http://hdl.handle.net/10138/332477

https://doi.org/10.1007/978-3-030-14741-9_5

unspecified

acceptedVersion

Downloaded from Helda, University of Helsinki institutional repository.

This is an electronic reprint of the original article.

This reprint may differ from the original in pagination and typographic detail.

Please cite the original version. 


\section{Chapter 4 - Membrane-containing Icosahedral Bacteriophage PRD1: The Dawn of Viral Lineages}

\section{Hanna M. Oksanen ${ }^{1}$ \& Nicola G. A. Abrescia ${ }^{2,3}$}

1 Molecular and Integrative Biosciences Research Programme, Faculty of Biological and Environmental Sciences, University of Helsinki, Viikinkaari 9, 00014 Helsinki, Finland.

E-mail: hanna.oksanen@helsinki.fi

2 Molecular Recognition and Host-Pathogen Interactions Programme, CIC bioGUNE, CIBERehd, Bizkaia Technology Park, Bld.800, 48160 Derio, Spain.

3 IKERBASQUE, Basque Foundation for Science, 48013 Bilbao, Spain

E-mail: nabrescia@cicbiogune.es

Keywords:

Viral lineage, PRD1, adenovirus, 3D structure, bacteriophage, picornaviridae, assembly, packaging, DNA ejection, uncoating 


\section{Abstract}

Membrane-containing enterobacterial phage PRD1 was isolated from sewage more than forty years ago. At the time none would have expected the impact that unravelling its biology would have on modern virology and on the way we understand virus assembly, evolution, and classification today. PRD1 structural analyses have provided a framework for understanding some aspects of virus evolution introducing the concept of 'viral lineages' - where the three dimensional structures of virus capsids represent the fingerprint for evolutionary relationship which cannot be traced from the sequence data. In this review we summarise those findings that have led to the concept of viral lineages and the multidisciplinary efforts made in elucidating its life cycle and that have rendered PRD1 a model system not only for the family Tectiviridae to which it belongs, but more generally to complex doublestranded DNA viruses enclosing a membrane vesicle beneath the capsid shell. 


\section{Introduction}

The first membrane-containing bacteriophage was isolated from the coastal sea water of Viña del Mar, Chile (Fig. 1A). The first identification of a bacteriophage that contains a lipid was the description of PM2 by Espejo and Canelo 50 years ago (Espejo and Canelo, 1968). They were inspired by a previous work by Spencer (Spencer, 1963), which reported that two bacteriophages active against marine pseudomonads were inactivated by organic solvents. Espejo and Canelo, motivated by this work isolated several strains of marine pseudomonads from the Pacific Ocean off the coast of Chile. A bacteriophage that grew on one of the strains was found in the same water and was named PM2. PM2 was found to be sensitive to ether, chloroform, and $0.05 \%$ Sarkosyl. Chemical analysis demonstrated that virions possessed DNA containing phosphorus, as well as another phosphorus-containing component that could be extracted with chloroform-methanol. This material proved to be phospholipid based on thin-layer chromatography.

A

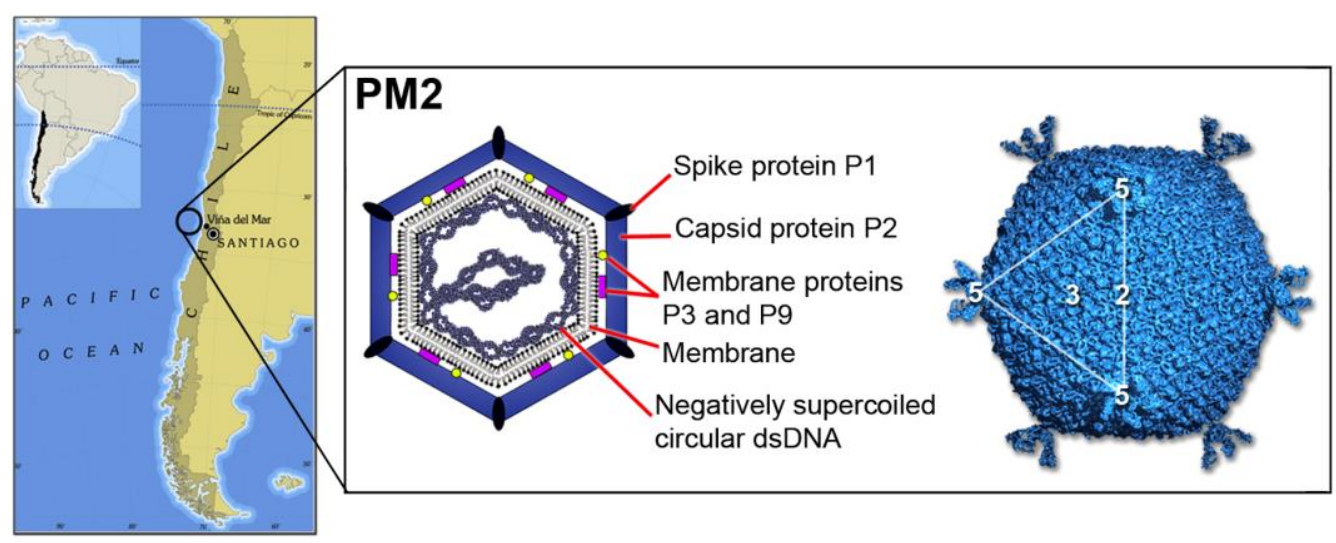

B

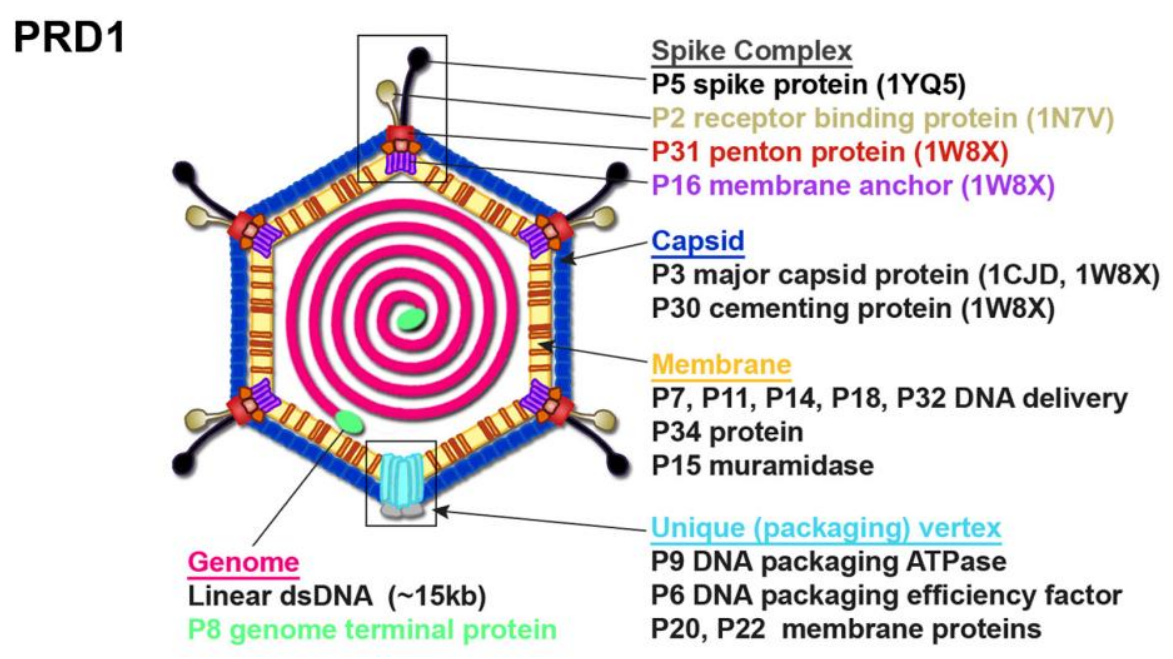


Fig. 1: First membrane-containing viruses studied.

A) Location where the first membrane-containing bacteriophage PM2 was identified and that paved the way to further studies on this type of viruses (Map: J. Oksanen). Inset, left, schematic representation of PM2 with its architectural components (Kivela et al., 2002); right, PM2 electron density map (blue) displayed at $1 \sigma$ determined by X-ray crystallography at $7 \AA$ resolution (Abrescia et al., 2008). The white triangle marks a virus facet, and numbers define icosahedral symmetry axes. $B$ ) Schematic representation of membrane-containing bacteriophage PRD1 (numbers in parentheses identify the corresponding protein structures in the Protein Data Bank) adapted from (Peralta et al., 2013); reproduced under the terms of Creative Commons Attribution.

Fifty years after its discovery, bacteriophage PM2 which infects marine gramnegative Pseudoalteromonas strains, is still the only member of the family Corticoviridae, although sequences related to PM2 are widespread in the genomes of aquatic proteobacteria (Oksanen and Ictv Report, 2017). Very recently phage Cr39582 was induced from Pseudoalteromonas sp. isolated from the gut of marine invertebrate Ciona robusta (Leigh, 2018). Cr39582 shows significant sequence similarity and gene synteny to PM2 proposing that Cr39582 could be the second member of the family Corticoviridae (Leigh, 2018). PM2 is a virulent phage with a supercoiled circular double-stranded (ds) DNA genome of $\sim 10 \mathrm{~kb}$ that is enclosed by the internal membrane vesicle located underneath the icosahedral protein coat (Abrescia et al., 2008; Kivela et al., 1999) (Fig. 1A, inset).

Discovery of the marine bacteriophage PM2 opened up a new research field and more membrane-containing phages were isolated in the early 1970s. One such phage was PRD1, isolated from Kalamazoo, Michigan (USA) sewage, which is the representative member of the family Tectiviridae (Olsen et al., 1974) (Abrescia et al., 2004) (Oksanen, 2012). The current understanding of PRD1 is shown schematically in Fig. 1B, left. It infects Gram-negative bacteria such as Salmonella enterica, Pseudomonas aeruginosa, and Escherichia coli that contain a conjugative plasmid belonging to the incompatibility (Inc) group IncP, IncN or IncW. The plasmid is essential for the phage as it codes for the receptor (Olsen et al., 1974). Tectiviruses have a tail-less, icosahedrally symmetric capsid approximately $65 \mathrm{~nm}$ in diameter, surrounding a proteinaceous membrane vesicle which in turn encapsulates the linear, dsDNA genome of approximately $15 \mathrm{~kb}$ (Oksanen, 2012). Viruses of this morphology also infect Gram-positive bacteria e.g. among the Bacillus cereus group, and this group of tectiviruses is genetically more diverse than the tectiviruses of gramnegative hosts (Gillis and Mahillon, 2014). Recently, tectiviruses were split into two 
genera Alphatectivirus and Betatectivirus where virulent PRD1-like tectiviruses belongs to the genus Alphatectivirus.

Two primary characteristics of PRD1 draw considerable interest for its use as a model system. Firstly, the PRD1 genome is replicated by a protein-primed, slidingback mechanism (Caldentey et al., 1992). The inverted terminal repeats on the genome and a covalently bound terminal protein at the 5 ' ends play a significant role in the replication of the linear dsDNA molecule (Bamford et al., 1983) (Savilahti and Bamford, 1993). Secondly, the membrane vesicle as part of the virion structure makes PRD1 a remarkable system to study the function and structure of the membrane vesicle during the virus life cycle (Abrescia et al., 2004; Azinas, 2018; Cockburn et al., 2004; Hong et al., 2014; Laurinavicius et al., 2007; Peralta et al., 2013; Sántos-Pérez et al., 2017). The well-defined genetic background, where PRD1 grows e.g. E. coli and S. enterica, together with a wide range of tools and the library of PRD1 mutants with optimized virus production and purification protocols (Bamford and Bamford, 1991; Mindich et al., 1982) have made PRD1 one of the most used membrane-containing virus models (Eskelin et al., 2016; Oksanen et al., 2012). In addition, the extensively studied assembly of PRD1 utilizing pre-assembled procapsids provides a framework for packaging and assembly of other large, complex icosahedral viruses with an internal membrane (Abrescia et al., 2004; Cockburn et al., 2004; Hong et al., 2014; Karhu et al., 2007; Peralta et al., 2013; Ziedaite et al., 2009) (Fig. 1B).

A significant step was taken in 1999, when the PRD1 major capsid protein (MCP) structure was solved and soon after that the shared conservation in the capsid structures between the phage PRD1 and human pathogenic adenovirus was recognized (Benson et al., 1999) (Fig. 2A). It was later observed that the MCP fold of PRD1 resembles that found in MCPs of certain other viruses infecting eukaryotes and archaea, possibly signifying descent from a common ancestor. The structurebased virus phylogeny based on the conservation of the MCP folds has been formulated and nowadays can be applied to a number of viruses irrespective of the virus family they represent (Abad-Zapatero et al., 1980; Bamford et al., 2002; Benson et al., 1999; Hogle et al., 1985; Nandhagopal et al., 2002; Rice et al., 2004; Rossmann et al., 1985). Here, we summarize those findings on PRD1 that have led to the concept of viral lineages and the most recent results that allow a deeper understanding of PRD1 life cycle. 
A

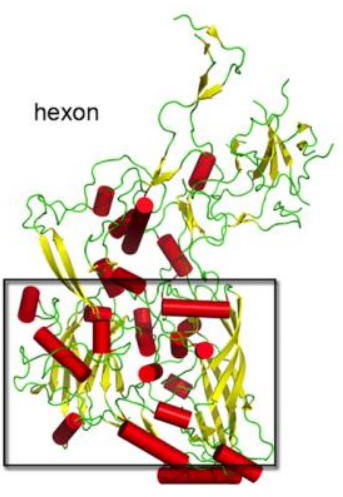

B
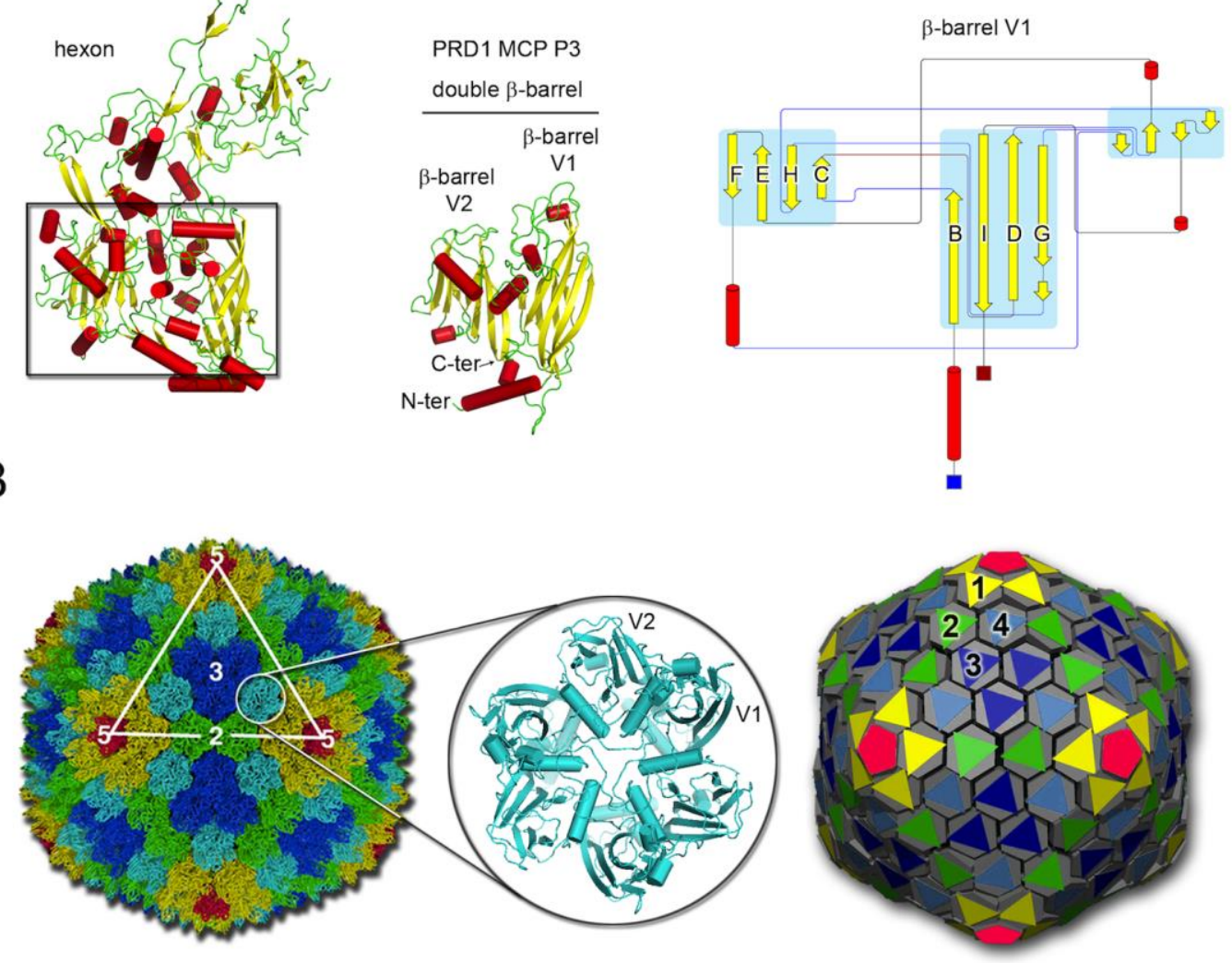

C
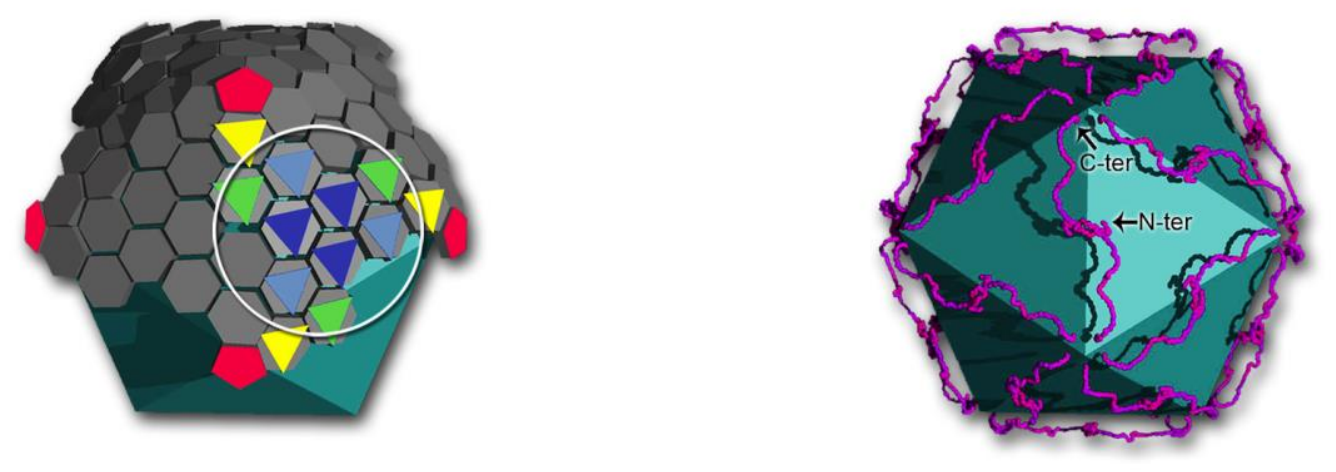

Fig. 2: PRD1 structure and assembly.

A) Side-by-side comparison of the MCP hexon in adenovirus (PDB ID 1P2Z) (left) with the MCP P3 in PRD1 (PDB ID 1CJD) (right) displayed in cartoon with a-helices represented as red cylinders and the conserved core double $\beta$-barrel as strands in yellow; the black rectangle on the hexon marks the double $\beta$-barrel. V1 (residues 15-245) and V2 (residues 246-383) define the individual $\beta$-barrel in P3; at the far right, the topological drawing of the V1 barrel (strands labelled as BIDG/CHEF) with the darkblue and red squares being respectively the $\mathrm{N}$-terminus and $\mathrm{C}$-terminus of $\mathrm{V} 1$ (http://munk.csse.unimelb.edu.au/pro-origami/). B) Left PRD1 atomic model as derived by crystallographic studies and showing the capsid organization represented with each of the four P3 MCPs trimers composing the icosahedral asymmetric unit (IAU) in ribbon and coloured in green, cyan, blue, and yellow; in red is the vertices penton protein P31 and white triangle indicates a virus facet with symmetry axes (PDB ID 1W8X) (Abrescia et al., 2004); adapted from (Peralta et al., 2013). The 
inset shows the 'cyan' P3 trimer as cartoon forming the capsomers viewed along the pseudo three fold axis. Right, geometric representation of PRD1 derived from the coordinates of the atomic model with solid hexagons (grey) to represents the pseudo-hexameric geometry adopted by the capsomers - the building blocks of the protein shell - which in turn are P3 trimers whose oligomerization is represented by flat triangles coloured as $(B)$; numbers 1-4 label the four independent capsomers of the IAU. C) Left, as $(B)$ right, but rotated $90^{\circ}$ clockwise with the bottom half of the capsomers removed showing the membrane underneath as light-cyan icosahedron and with a white circle defining the group-of-nine (GON) capsomers composing the planar virus facet as in adenovirus. Right, all caposmers have been removed so the tape measure P30 protein (83 residues) is visible (magenta); P30 stretches linearly from the $\mathrm{N}$-terminus located at the icosahedral 2-fold to the C-terminus located at the icosahedral 5fold and it dimerizes at 2-fold so two P30 molecules cement the edge of a triangular virus facet.

\section{Assembly of PRD1 and the tape measure P30}

Over the years the devised optimized protocols in production and purification of PRD1, and derived specific particles (e.g. mutant particles or subviral complexes) together with the advances in cryo-electron microscopy (cryo-EM) techniques made possible its structural investigation. In 1995, the first three-dimensional (3D) visualisation of the whole PRD1 particle was obtained by cryo-EM at $28 \AA$ resolution (Butcher et al., 1995). This structure illustrated the position of the MCPs P3 and hinted at the importance of the interactions between the membrane vesicle and the capsid layer. Importantly, it was only after the crystal structure of P3 at $1.85 \AA$ was resolved some years later in 1999 that the comparison was done with the crystal structure of adenovirus type 2 hexon (Athappilly et al., 1994) (Benson et al., 1999); the unexpected finding that both MCP possessed a similar fold prompted further cryo-EM analyses. A quasi-atomic model of PRD1 was achieved by fitting the P3 crystal structure into improved cryo-EM maps of PRD1 which lead to new information about the contacts across the MCPs building the virus facet and the membrane vesicle underneath (Benson et al., 1999; San Martin, 2001). Soon after, in 2002, the PRD1 architecture was analysed using difference imaging between several cryo-EM reconstructions (at $\sim 13 \AA$ resolution) and their quasi-atomic models (San Martin et al., 2002). Integration of functional studies with analysis of the viral mutant particles and difference maps identified not only small proteins at the capsid edges and how the peripentonal P3 MCPs connect to the vertex complex, but also the contacts cementing the capsid and linking it to the membrane.

Despite the advances in the understanding of the PRD1 structure it was not until its X-ray structure was determined at $4.2 \AA$ resolution that the full network of protein 
interactions and the principles governing the PRD1 assembly became clear (Abrescia et al., 2004) (Cockburn et al., 2004). PRD1 was the first - and it remains still today the only - virus with an internal membrane to have been studied by X-ray crystallography and solved at high resolution. The crystal structure of PRD1 revealed the molecular contacts that hold together the 720 copies of the MCP P3 on the membrane vesicle and the membrane structure with its interactions with viral proteins and the dsDNA (Fig. 2B). Four P3 trimers (capsomers) compose the viral icosahedral asymmetric unit (Nos 1-4 in Fig. 2B). Capsomers 2-4 arrange in the so-called group of nine (GON), as originally defined for the hexons in adenovirus and studied by negative stain EM, laying out the virus central facet with a strikingly similar architecture to adenovirus (both viruses possess the same triangulation number, pseudo $T=25$ (Figs. 22B and 22C, left) (Abrescia et al., 2004) (Liu et al., 2010).

PRD1 scaffolding protein P30, stretching linearly, cements the edges of the icosahedron and sizes the virion (Fig. $2 \mathrm{C}$, right). The eleven of twelve vertices are capped by peripentonal P3 trimers and by spike complexes composed of vertexstabilizing membrane protein $\mathrm{P} 16$, penton protein $\mathrm{P} 31$, spike protein $\mathrm{P} 5$, and receptor binding protein P2 (Huiskonen et al., 2007) (Jaatinen et al., 2004) (Xu et al., 2003) (Figs. 21B and 22B, left and centre). The internal membrane enclosing the densely packaged dsDNA - up to four spooled layers are visible - is composed of phosphatidylethanolamine (PE) and phosphatidylglycerol (PG) lipids and membrane proteins at roughly equal mass (Cockburn et al., 2004). The two lipid species are asymmetrically distributed across the membrane bilayer, with PG enriching the outer leaflet while the PE the inner leaflet (Cockburn et al., 2004). At long last, recent studies (see Section-4) have structurally identified and elucidated the unique vertex, which is the twelfth vertex where the membrane-embedded portal is used for DNA packaging into the procapsid (Gowen et al., 2003; Hong et al., 2014; Stromsten et al., 2003). This unique vertex also serves as a conduit for DNA ejection through the proteo-lipidic tube formed from the internal protein enriched membrane vesicle (Peralta et al., 2013). In summary the elucidation of the principles governing the assembly of PRD1 scalable to other viruses opened the door to a new conceptual framework for virus taxonomy that has allowed to establish viral evolutionary relationships otherwise undetectable. 


\section{The dawn of viral lineages}

Sequence homology is easily lost when comparing viral sequences, even across viruses that belong to the same family. The earliest structural analyses of viruses, however, showed that the capsid protein fold and the arrangement of protein subunits can be preserved between viruses that belong to different families and infect divergent hosts. Some of the first plant and animal virus structures (family Picornaviridae) revealed prominent similarities in their MCP folds which was assumed as probably demonstrating a descent from a common ancestor regardless of any noticeable sequence similarity (Abad-Zapatero et al., 1980; Hogle et al., 1985) (Rossmann et al., 1985). These MCPs displayed single horizontal $\beta$-barrels and arranged with the long axis of the $\beta$-barrel tangentially to the virus surface. At the time, the paucity of available virus structures hindered the understanding of which similarities were the remains of an ancestral relationship from those that appeared simply because there was no other way of forming a suitable viral capsid. Fifteen years passed before it was shown that the enteric membrane-containing bacteriophage PRD1 and human adenovirus share a common coat protein fold, the so-called double $\beta$-barrel jelly-roll (or double jelly-roll) (Benson et al., 1999) (Athappilly et al., 1994) (Fig. 2A).

Both PRD1 MCP P3 and adenovirus hexon arranged in similar trimers, with the long axis of the $\beta$-barrels orthogonal to the virus surface, to form pseudo-hexagonal capsomer structures, the elemental building blocks of the icosahedral facets from which both of these virions are constructed (Fig. 2B, C). This observation led to the proposal that viruses could be grouped in viral lineages using the structural similarity of their MCPs, and precipitated a re-analysis of the low resolution structure of the chlorella virus PBCV-1 (family Phycodnaviridae), one of the largest viruses known at the time (Bamford et al., 2002). A simple and yet perspicacious manual fitting of the four P3 capsomers making up the PRD1 icosahedral asymmetric unit into the 3D PBCV-1 reconstruction supported the hypothesis that it belonged to the same lineage (Fig. 3A) (Bamford et al., 2002). Few months later the crystal structure of PBCV-1 MCP Vp54 determined at $2 \AA$ resolution provided the experimental proof to the groundwork layout by Bamford and colleagues (Bamford et al., 2002; Nandhagopal et al., 2002). 
A
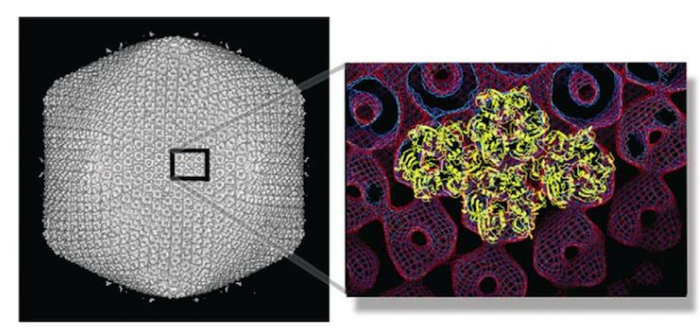

B

PRD1 like viral lineage

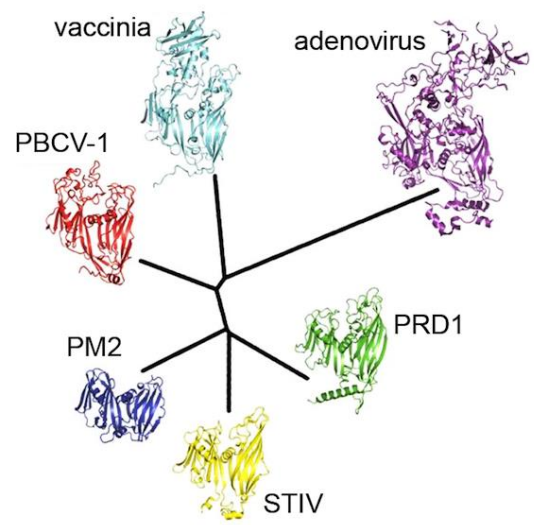

C

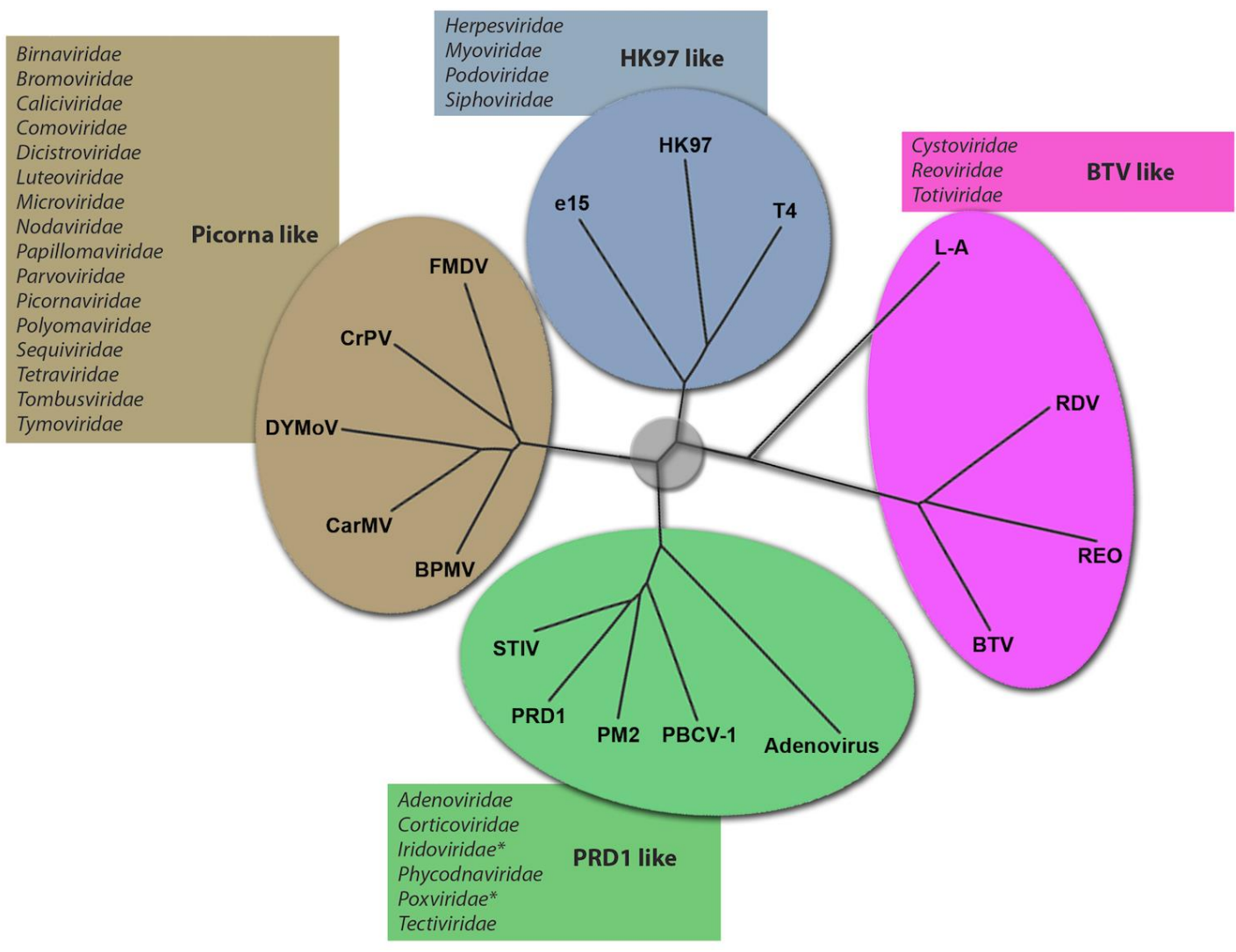

Fig. 3: Structure-based viral lineages.

A) Surface rendering of the 3D reconstruction of PBCV-1 at $\sim 26 \AA$ resolution (Yan et al., 2000) with a preliminary fit of the atomic structure of the PRD1 MCPs P3 into the PBCV-1 reconstruction anticipating some of the structural assembly principles of the PRD1-like viral lineage, adapted from (Bamford et al., 2002). B) PRD1-adeno structure-based phylogeny tree of the MCPs of vaccinia (D13), PBCV-1, adenovirus, STIV, PRD1 and PM2. MCP atomic models are represented in cartoon and differently coloured. Fig. 3 reproduced from (Bahar et al., 2011). C) Structure-based phylogenetic tree showing the four different lineages identified by structural comparison of viral MCPs. Details of the methods and atomic models used are described in (Abrescia et al., 2012) from where the Fig. 3 has 
been reproduced with permission. The table associated to each clade lists the virus families (left) of the virosphere that can be placed in the corresponding viral lineage (right).

This knowledge, together with the crystal structure of the whole PRD1 that allowed the understanding of its assembly pathway and offered a unique scalable mechanism for determining particle size by using a molecular tape measure, prompted the possibility that many more viruses within the virosphere could belong to the same PRD1-adeno viral lineage (Abrescia et al., 2004). At the time the structural sampling of viral MCPs and/or viruses represented, however, (and regardless of all subsequent efforts - still represents) a very small fraction of the enormous amount of viruses, $10^{30}-10^{31}$, that have been estimated to permeate the biosphere (Suttle, 2007). Despite this astronomical number, whether viruses could be defined by few structural principles - recapitulating the building of the viral capsid and the encapsidation of the viral genome - came into question. Topology alterations in proteins in any of these processes would be disadvantageous as possibly compromising the virus identity 'self' - and/or progeny. Since 2002 several more structures of viral MCPs and viruses have been determined by different laboratories worldwide thus increasing the conformational sampling of the MCP topology adopted by viruses (Abrescia et al., 2008; Baker et al., 2005; Jiang et al., 2003; Khayat et al., 2005; Yan et al., 2009; Zhang et al., 2011). Viruses (e.g. PBCV-1, STIV, PM2) infecting hosts residing in all three domains of life were identified as possessing a MCP with a double $\beta$-barrel fold and expanding the PRD1-adeno lineage (Fig. 3B). This structural similarity between proteins seen in viruses infecting their own hosts representing all three domains of life suggested that this is a very ancient lineage, whose origins predate the separation of the domains.

In 2012, a systematic structure-based analysis across all available virus coat protein folds provided a new means to classify viruses within the viral universe (virosphere) (Abrescia et al., 2012). Such study shows the existence of four different lineages for icosahedral viruses presently identified (Fig. 3C). The PRD1-adenovirus lineage is now postulated to include six virus families (Fig. 3B). Strikingly, pleomorphic vaccinia viruses (family Poxviridae), through viral protein D13 possessing the double $\beta$-barrel fold, share the pseudo-hexagonal structural building block characteristic of this lineage; albeit D13 is used only transiently as a scaffold during the virion assembly (Bahar et al., 2011). 
There is no doubt that only a tiny fraction of viruses have been identified so far and even a smaller number has been structurally analysed. Consequently, new virus coat protein folds will be discovered in the future. Nevertheless the systematic comparison and classification of all currently known virus coat protein folds - and virion architectures when available - represent a powerful means to establish relationships across members of the virosphere apparently not related; and this will remain so at least until the sensitivity of current standard bioinformatics tools for detection of sequence similarity is improved (Sinclair et al., 2017).

\section{PRD1 genome packaging and delivery}

The studies on PRD1 assembly fuelled also the investigation of other aspects of the PRD1 lifecycle that remained obscure still. One of the fundamental steps in any virus lifecycle is the packaging of the genome. In icosahedral dsDNA bacteriophages the genome is translocated into preformed procapsids using specific enzymes, terminases or packaging ATPases (Lokareddy et al., 2017; Hong et al 2016 PRD1). In some bacteriophages the elastic, electrostatic, entropic, osmotic, and hydration energetic costs incurred by constraining the dsDNA at very high densities within the fixed volume of the capsids build up a significant internal pressure (Molineux and Panja, 2013). This pressure is considered to provide the force initially driving genome injection into the cell, while DNA-binding proteins, DNA condensation or enzymes may help during the final stages of the transfer (Jeembaeva et al., 2010). In the tailed phages (order Caudovirales) the DNA packaging occurs at a unique portal vertex. In the mature virion this special vertex is equipped with a pre-formed proteinaceous tail which is used as a DNA ejection device during infection. Several bacteriophage portals to which the tail attaches during assembly have been studied. One of the best characterised packaging systems is that of $\Phi 29$, a short-tailed icosahedral virus with a prolate capsid containing a linear dsDNA genome, infecting Gram-positive bacteria (Simpson et al., 2000) (Mao et al., 2016). Enterobacterial phage P22 portal has also been analyzed in great biochemical and structural detail recently leading to the elucidation of the mechanism that couples genome packaging with capsid maturation (Lokareddy et al., 2017). A portal assembly complex has also been identified in herpes simplex virus type 1 (HSV-1), a eukaryotic enveloped virus whose nucleocapsid forms an icosahedral protein shell. The portal of HSV-1 is located at a single vertex of the nucleocapsid and contains a ring-shaped multimer of the UL6 protein (Newcomb et al., 2001) (Cardone et al., 2007). 
In the case of tail-less PRD1 several efforts have been made to understand how the dsDNA is packaged in these types of viruses that possess a further architectural element constituted by the protein-rich membrane vesicle, and how the dsDNA is ejected into the host-cell at infection. In particular as early negative stain 2D images of PRD1 showed the occasional presence of a tubular appendix protruding from one of the twelve icosahedral vertices, a tubular structure that was also visualised by analysing the isolated viral membrane vesicles (Bamford and Mindich, 1982). The first confirmation of a unique vertex in PRD1 came from immune-gold negative-stain experiments where gold-conjugated antibodies against proteins P6 and P20 were shown to bind to a single vertex of PRD1 (Gowen et al., 2003). This suggested that at least the minor capsid protein P6, which has no previously appointed function, and the membrane protein P20 are located at a unique vertex. Biochemical and genetic studies were also performed to dissect both the genome packaging and ejection mechanisms (Grahn et al., 2002a). These studies revealed two sets of proteins respectively involved in DNA packaging (or in the stable maintenance of the DNA within the particles) and in the DNA delivery system. The former were small membrane proteins $\mathrm{P} 20$, P22, the packaging efficiency factor P6, and the packaging ATPase P9 and the latter ones were proteins P7, P14, P11, P16, P18, and P32 (Grahn et al., 2002a)(Grahn et al., 2002b; Karhu et al., 2007) (Hong et al., 2014). The packaging ATPase P9 powered by ATP hydrolysis, pumps the dsDNA into the empty procapsid; to harness this mechanism an in vitro system for packaging DNA into preformed procapsid was developed (Stromsten et al., 2005).

The averaging techniques used to investigate the 3D PRD1 structure while provided exquisite details of icosahedrally ordered protein components, they blurred all those that do not obey icosahedral symmetry. Only when cryo-EM single particle asymmetrical reconstruction was used the location and the structure of the unique vertex were determined (Hong et al., 2014). The special vertex is occupied by several protein components not obeying to the 5-fold symmetry (Fig. 4A). Ten MCP P3 trimers wrap around the 12-fold symmetrical P9/P6 complex at the unique vertex (Figs. 24A inset and 24B). Such a symmetry mismatch is also a structural hallmark of the tailed dsDNA viruses with a portal complex arranged with 12-fold symmetry (Lokareddy et al., 2017). Strikingly, the capsid and the icosahedral internal membrane vesicle interconnect via membrane proteins P20 and P22, organized as 6- 
fold symmetry, to the P9/P6 complex portal, organized as 12-fold symmetry, thus generating a symmetry mismatch (Fig. 4B).

A

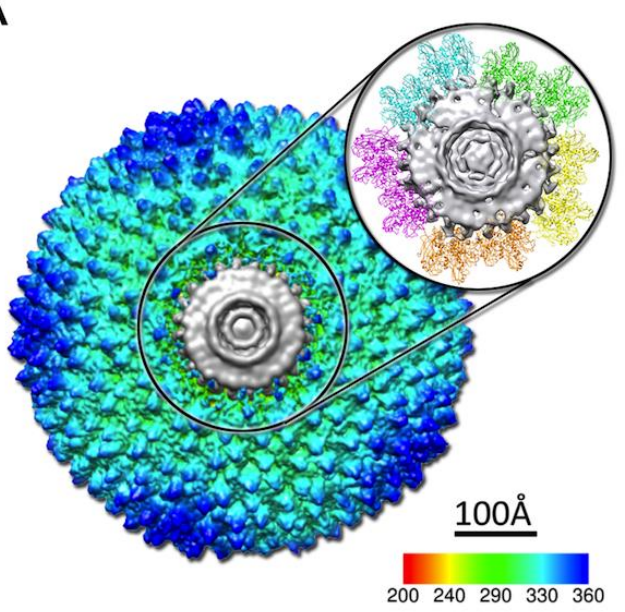

C
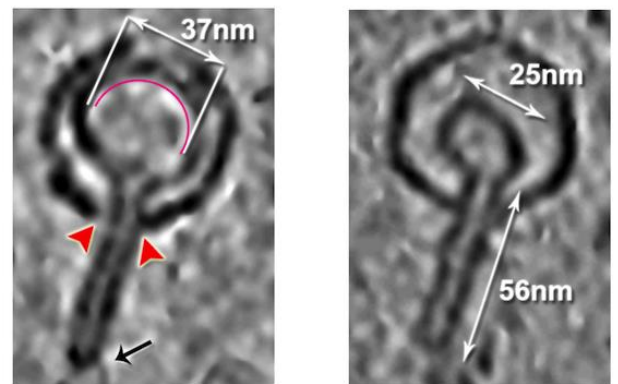

B

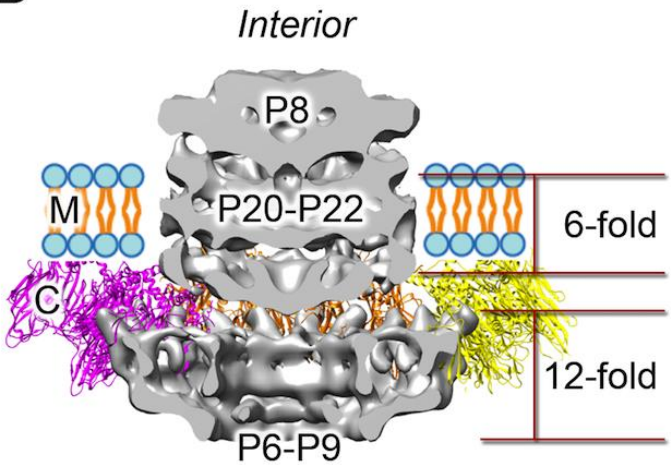

Exterior

D

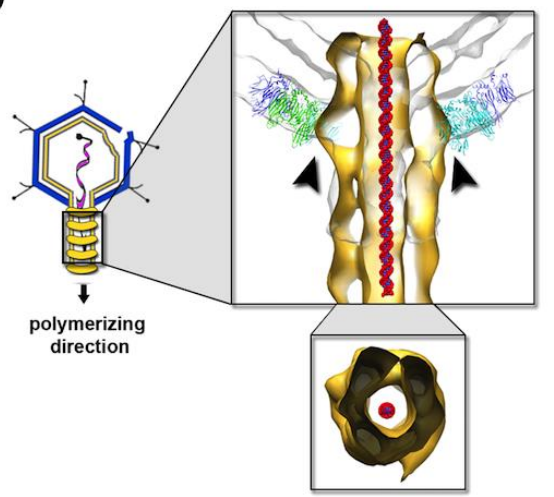

$E$
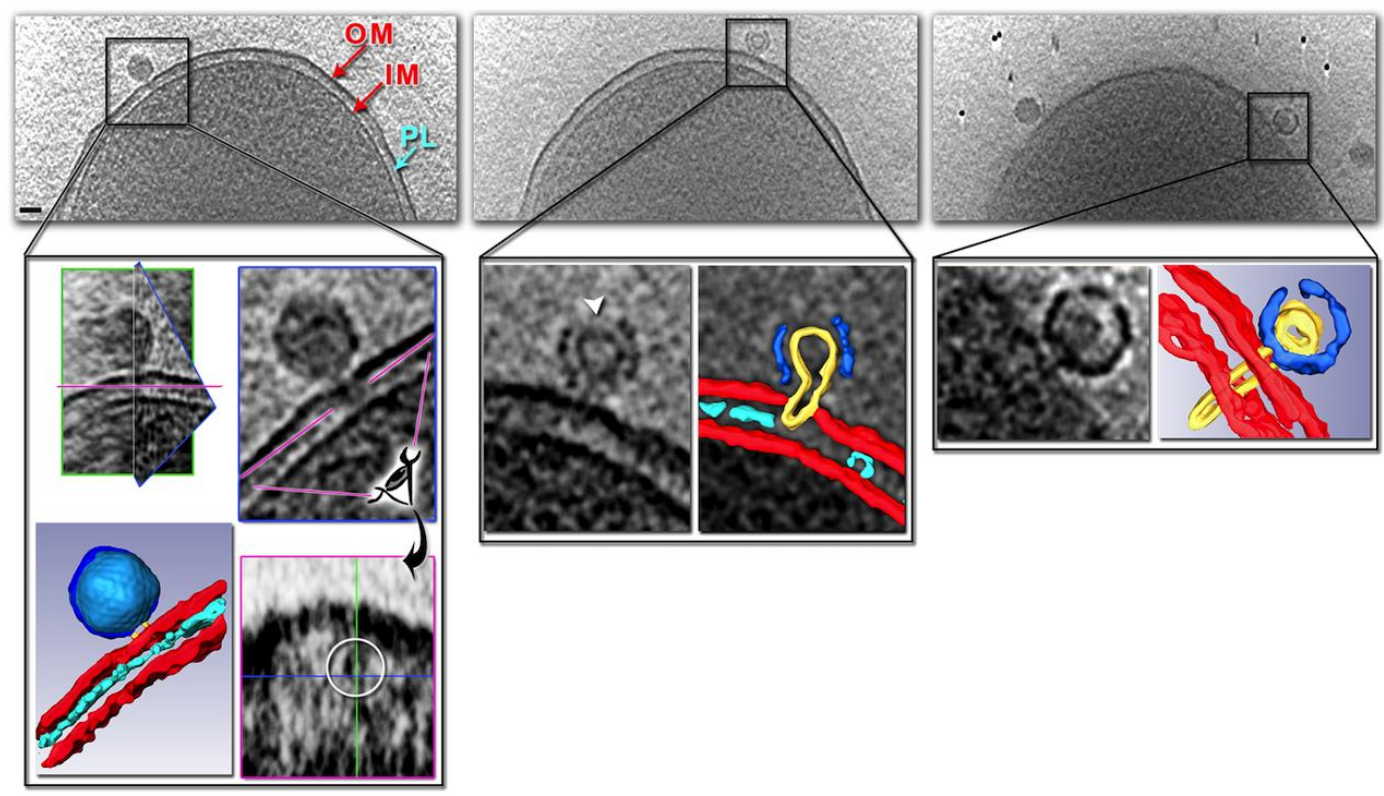

Fig. 4: PRD1 unique vertex and DNA ejection mechanism.

A) Surface rendering of the asymmetrical 3D reconstructed cryo-map of PRD1 at $12 \AA$ resolution viewed along the symmetry axis of the unique vertex (grey) and coloured by radius (colour bar); right 
top inset shows the 10 MCP P3 trimers (represented as cartoon and coloured in green, yellow, orange, magenta and light-blue) wrapping around the 12-fold symmetrical P9/P6 complex at the unique vertex. B) cross-sectional view of the architecture of the unique vertex coloured as in (A), $M$ marks the membrane depicted schematically, and $C$ the capsomers surrounding the unique vertex. The unique vertex is modular and composed of the DNA terminal protein P8, of the membrane embedded P20/P22 complex forming the 6-fold symmetrical membrane conduit for the portal vertex, and, on the exterior, of proteins P6 and P9 assembling in a 12-fold symmetrical complex. (A-B) adapted from (Hong et al., 2014) under the terms of Creative Commons Attribution. C) Left, tomographic central xy section of a PRD1 particle possibly at initial stage of DNA ejection with a tail tube self-assembled and the internal membrane vesicle not yet fully deflated; the semicircular red line within the vesicle marks the possible presence of residual genome whereas the red arrowheads indicate the vertex aperture from where the tube protrudes and the black arrow the conical tip of the tube. Right, same as left but a different PRD1 particle with the tube possibly at the final stages of the DNA ejection with the vesicle showing a "map pin" morphology. D) Schematic of PRD1 with tube with inset showing as cut-through the sub-tomogram averaged tube density (in gold) within the capsid region corresponding to the vertex from where the tube protrudes (semitransparent light-grey); the black arrowheads mark a ring-like structure visible in one of the tube averaged classes and that likely correspond to peripentonal P3 MCPs surrounding the unique vertex (Hong et al., 2014) (see also B); inset below shows an orthogonal view of the tube density, a B-form dsDNA is modelled in the tube channel. E) Cellular tomographic slices of an E.coli cell infected by PRD1. Scale bar, $50 \mathrm{~nm}$. Left, full PRD1 particle, outlined by a black square, with forming tube crossing the outer membrane (OM), inner plasma membrane (IM) and peptidoglycan layer $(\mathrm{PL})$ are indicated; the inset below shows on the lefttop the particle viewed through three intersecting planes (outlined in green, blue, and magenta with corresponding blue and magenta planes on the right while on the left-bottom a segmentation of the particle (dark-blue) is superimposed with the density derived from the atomic model (PDB code 1W8X) in light-blue, in red the outer and inner membranes, and cyan the peptidoglycan layer. Centre, semifull particle, outlined by a black square with below inset showing a tomographic slice (left) with superimposed a segmentation of the capsid and cell envelope (right); the white arrowhead defines additional openings of the capsid. Right, an almost empty particle with the inset below showing a particle with a "map-pin" shape vesicle and full-length tube penetrating the cell envelope (left) and corresponding segmentation (right). (C-E) reproduced from (Peralta et al., 2013).

Using an integrative approach combining immuno-labelling, cryo-EM and cryoelectron tomography (cryo-ET) techniques, and using mature PRD1, PRD1 procapsid (defective in P9) and in vivo analysis of virus-infected cells, Peralta and colleagues reported a new principle of virus-cell interaction essential for viral genome translocation (Peralta et al., 2013). The study anticipated that the PRD1 DNA ejection tube, captured many years earlier by negative-stain EM techniques, protrudes from the same unique vertex used for DNA packaging and that it is structured (Fig. 4C, D). These findings support the direct involvement of self-assembling and lattice forming membrane-associated proteins with likely candidates being P7, P14, P18, and P32 
proteins (Grahn et al., 2002a). Importantly, the packaged genome does not have a role in the triggering or in the formation of the tail tube. However, in the procapsid where the membrane vesicle is devoid of the genome, the formation of tail tube fails to make the correct orientation and consequently the tubular structure does not always protrude from the vertex. Moreover, the genomeless membrane vesicle displayed diverse morphologies from stomatocyte-like to discocyte-like revealing not only the elasto-mechanical properties of giant unilamellar (proteo-)vesicles but also the triggering factors leading to the remodelling of the membrane into the tail tube (Peralta et al., 2013). These factors were attributed to the change in structural and environmental and/or osmotic conditions of the internal membrane vesicle upon binding of the phage to the cellular receptor. This event (or multiple events) would initially alter the structure of the vertex complex leading to the de-capping of the vertices, thus exposing the membrane to the extra-cellular milieu conditions and initiating the remodelling of the membrane vesicle and tube self-assembly process. It has also been postulated that the membrane elastic properties could aid the translocation of the viral genome into the host-cell during the initial stages of infection (Sántos-Pérez et al., 2017). Finally, the use of cellular cryo-ET remarkably captured PRD1 infecting a E.coli cell in vivo using the tail tube to drill into the bacterial cell envelope, leaving no doubts as to the mechanisms governing the tube self-assembly, the viral DNA ejection apparatus and the cell infection (Fig. 4E) (Peralta et al., 2013).

\section{Mechanical properties of PRD1}

The coupling of the fold of the viral MCPs with the building principles of the virion, and the genome packaging mechanism does not only emerge as signature for detecting virus common ancestry but it is the same quintessential purpose for protection of genome integrity.

To secure the genetic cargo outside the cell, viruses have devised different viral protein folds, architecture and material composition (protein or lipids or a mixture of both). Indeed when travelling outside of the host cell, virions may encounter harsh environmental conditions. This combination of materials and architectures provide specific mechanical properties fulfilling the requirement for protection. Singlemolecule techniques such as atomic force microscopy (AFM) have been proven to be very useful in unravelling the mechanical properties of viruses (Martin-Gonzalez et al., 2018). This information is pivotal not only to grasp their biology but also for 
manipulating and using them as nano-carriers of foreign cargos. AFM studies on viruses have provided unprecedented knowledge of the deformability (stiffness, $k$ ), the energy required for mechanical failure (toughness, $T$ ), and the limits to fatigue for virions (Llauro et al., 2016). However, the majority of these studies have been performed on icosahedral viruses with a proteinaceous capsid shell enclosing the genome (DNA or RNA).

Only recently, to the best of our knowledge, the first AFM study on viruses such as PRD1, where an internal membrane vesicle surrounds the densely packaged dsDNA, was performed (Azinas et al., 2018). The wealth of genetic, biochemical, and structural information on PRD1 together with the availability of different particles: mature PRD1, procapsid (devoid of DNA and packaging ATPase P9), P3-shell (icosahedral P3-shell composed of MCP P3 and minor capsid protein P30, but lacking the pentons and peripentonal capsomers), and proteo-lipidic membrane vesicle enclosing a complete genome, lend PRD1 an amenable system for AFM examination. The morphology and mechanical responses of these particles were assessed in an aqueous environment and their stiffness and yield behaviour under an applied force analysed (Fig. 5A-I). 

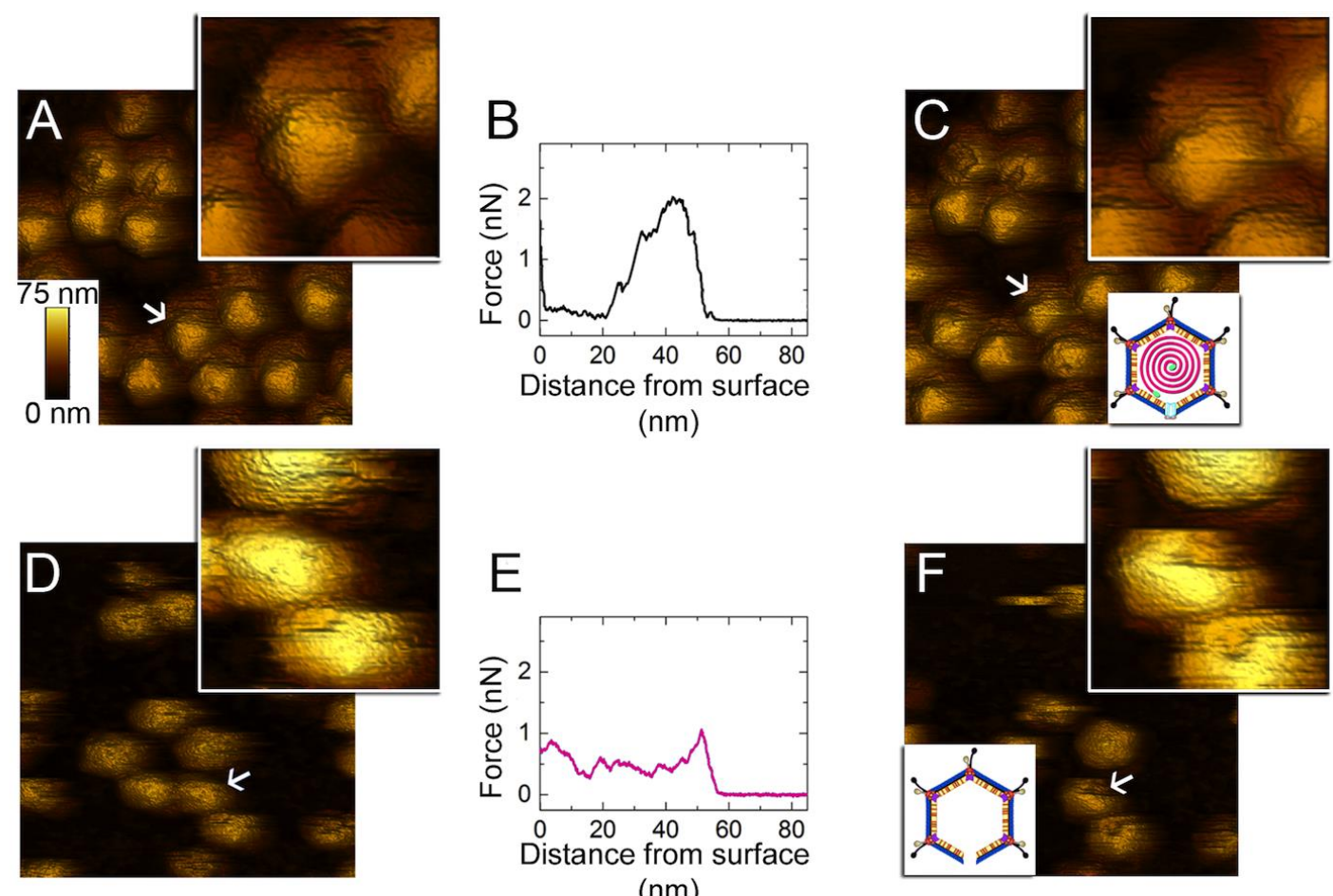

$(\mathrm{nm})$
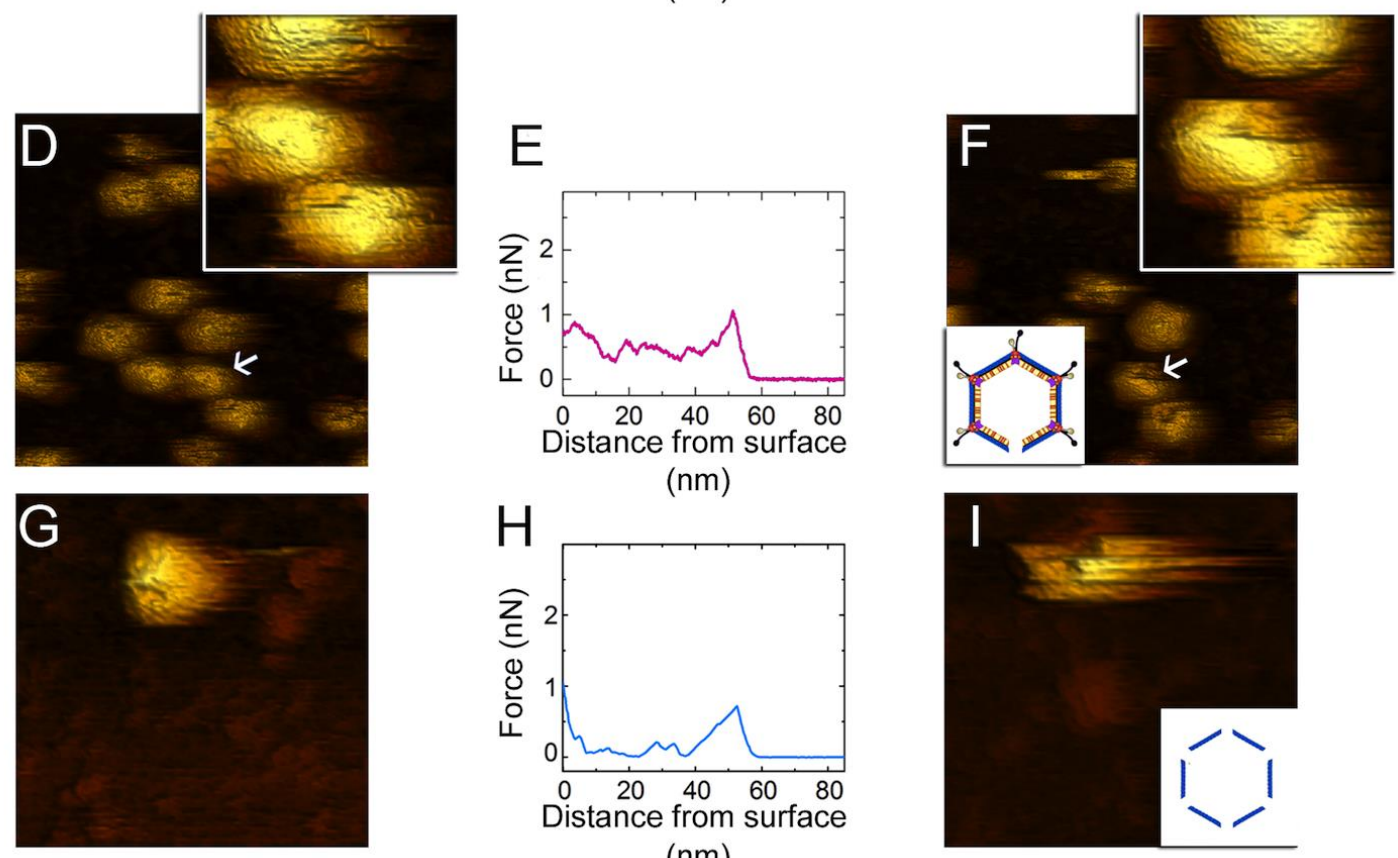

$(\mathrm{nm})$
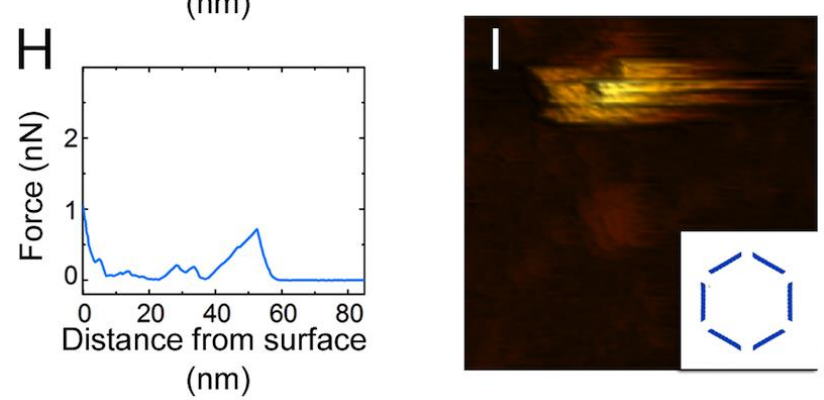

$\mathrm{L}$

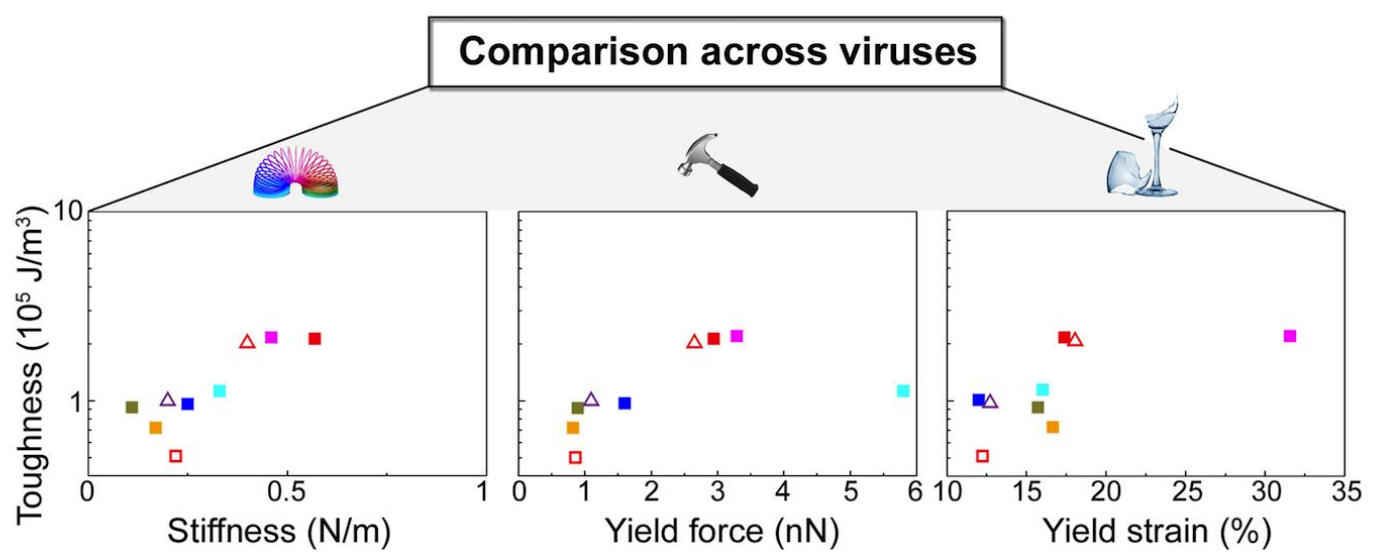

Fig. 5: The mechanics of PRD1.

Representative topographic consecutive AFM images deriving from nanoindentation studies of the different PRD1 particles with (A), (D), $(G)$ and $(J)$ before and $(C),(F)$, and $(I)$ after nanoindentation with schematics identifing particle types: wt PRD1 (top), Sus1 procapsid (middle) and P3-shell (bottom) (vesicle not shown; vesicles displayed the least stiffness and not defined yield points). Panels (B), (E) and $(H)$ represent a single mechanical indentation, with the corresponding force curve obtained for each particle type. Image size: $500 \mathrm{~nm}$; $z$ range (vertical dimension): $75 \mathrm{~nm}$, see color key in (A). The white arrows in $(A),(C),(D)$, and $(F)$ mark the indented particles with insets showing their view 
enlarged (image size: $160 \mathrm{~nm}$ ). (L) PRD1's mechanical properties compared to other dsDNA viruses via Ashby plots of toughness ( $y$-axis in log scale) vs. stiffness, yield force and yield strain ( $x$-axis), respectively, with the slinky, hammer and broken glass symbolising the investigated mechanical property. The filled, empty squares and empty triangles identify virions, penton-less particles and procapsids respectively; PRD1 particles in red, bacteriophage $\lambda$ in blue, HSV-1 in cyan, bacteriophage P22 in purple, adenovirus in magenta, bacteriophage T7 in orange and bacteriophage HK97 in darkgreen). Adapted from (Azinas, 2018).

One of the major findings is that PRD1 displays a similar breaking force whether it is packaged or devoid of DNA, with the presence of the DNA inducing a stiffening of the mature PRD1 over the procapsid (Azinas et al., 2018). This implies that the mechanical stability of mature PRD1 is independent from the presence of the genome. In contrast, the fragility (brittleness) of the P3-shell particle unequivocally demonstrates the decrease in structural reinforcement of the particle when only interactions across capsomers containing the GON and the cementing protein P30 are considered. The protein-rich membrane vesicle displayed the least stiffness and no clear yield point. Thus the contribution of the membrane to the overall stiffness of mature PRD1 is marginal, mainly transmitting the stiffening due to the DNA pressure to the capsid. Interestingly, when the architecture of PRD1 was modelled as a simplified two-shell composite capsid-membrane system using the experimentally derived mechanical properties, it was confirmed that the presence of the membrane layer contributes less than $10 \%$ to the stiffness of the particle (Azinas et al., 2018). To assess how the capsid and membrane vesicle together influence the resilience, the toughness was estimated for each PRD1 derived particles and compared with available dsDNA icosahedral viruses. Surprisingly, the toughness of the mature PRD1 is rivalled only by adenovirus (Fig. $5 \mathrm{~L}$ ). Adenovirus does not possess an internal membrane but a relatively large number of cementing proteins (IIla, V, VI, VIII and IX) that glue together the different hexons making up the capsid shell (Liu et al., 2010). Although deconvoluting the individual contribution of the capsid and membrane vesicle to the overall PRD1 toughness is challenging as an intact capsid cannot be produced, PRD1 mechanics indicate that the proteinaceous capsid is stiff and brittle, whereas the membrane vesicle is ductile and soft (Azinas et al., 2018). However, it is only when these two layers and materials are architecturally integrated that PRD1 achieves a high resilience. Thus, the mechanical properties displayed by the mature PRD1 are reminiscent of those of a composite material not man-made but optimized through evolution for the protection of the fragile and precious genome. 


\section{The appearance of single vertical $\beta$-barrel viruses}

In Section 3 we accounted for the origin of the concept of viral lineages based on structure and the efforts made from thereafter to provide a systematic classification of the virosphere. An increased interest in unexplored extreme ecological niches such as thermophilic and halophilic environments, led to the isolation of viruses displaying PRD1-adenovirus type architecture, but with two genes encoding two MCP types, each with a single $\beta$-barrel and both composing the lattice. We have called them "vertical single $\beta$-barrel viruses". The first isolate was highly halophilic virus $\mathrm{SH} 1$ of euryarchaeal host Haloarcula hispanica (Bamford et al., 2005). Other viruses of this group are thermophilic Thermus phages P23-77 and IN93, halophilic Salisaeta phage SSIP-1, and archaeal halophilic viruses HHIV-2, HCIV1 and PH1 infecting Haloarcula species and SNJ1 infecting Natrinema species (Aalto et al., 2012; Demina et al., 2016; Jaakkola et al., 2012; Jaatinen et al., 2008; Porter, 2013; Zhang et al., 2012). Currently, all such viruses are either bacteriophages or archaeal icosahedral viruses with an internal membrane and thriving in extreme surroundings and classified to the family Spherolipoviridae (Pawlowski et al., 2014). Only SSIP-1 is currently unclassified. Common to all vertical single $\beta$-barrel viruses is that they have a conserved gene that encodes for a putative packaging ATPase similar to those found in the double $\beta$-barrel viruses and shown in PRD1 to be the packaging ATPase at the portal vertex (Hong et al., 2014). In addition, virus-related, genome-integrated elements resembling the core elements of single $\beta$-barrel viruses have been identified in the genomes of bacterial Meiothermus and archaeal Halomicrobium and Haladaptus, Halociforma, and Haloarcula species suggesting that vertical single $\beta$ barrel viruses are more common than previously thought (Demina et al., 2016; Pawlowski et al., 2014; Porter, 2013) (Fig. 6). 

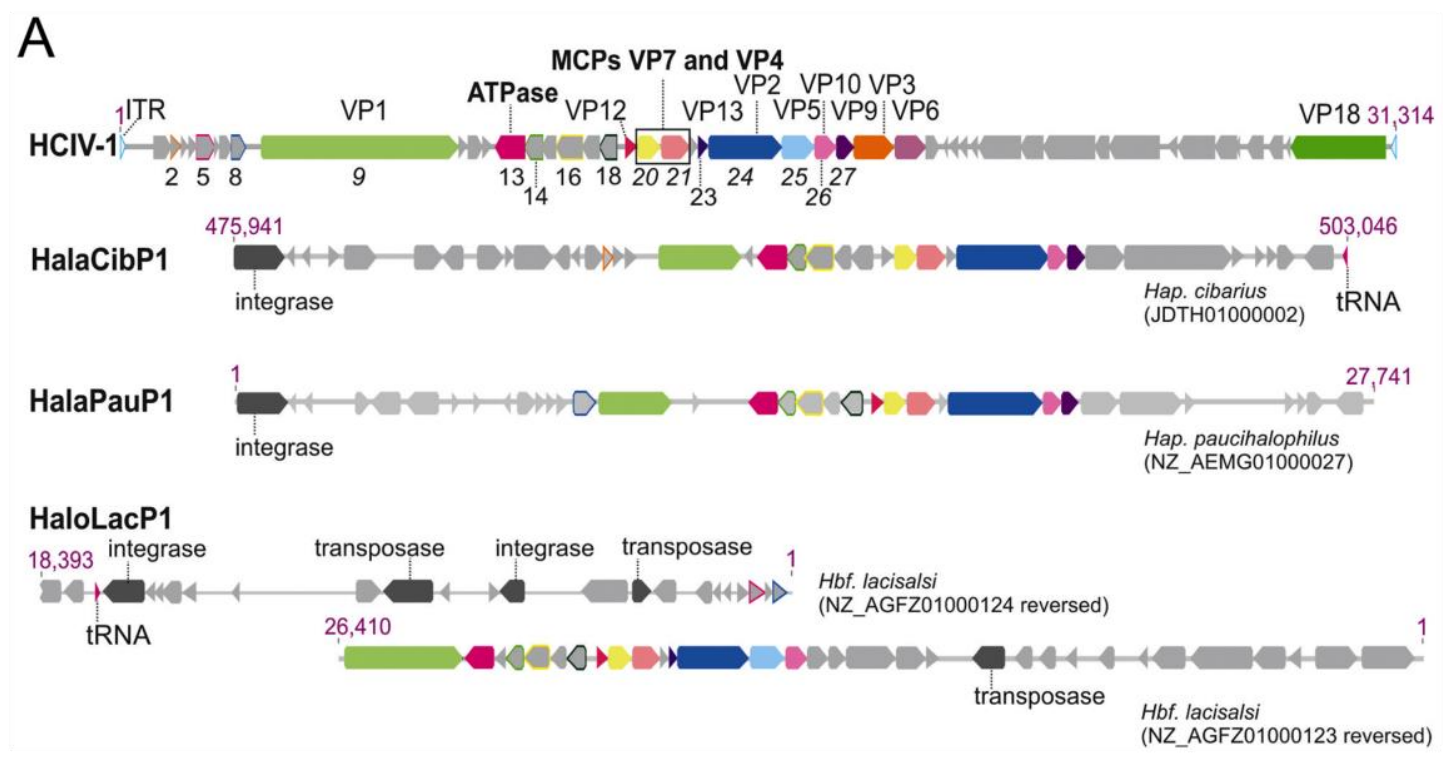

B

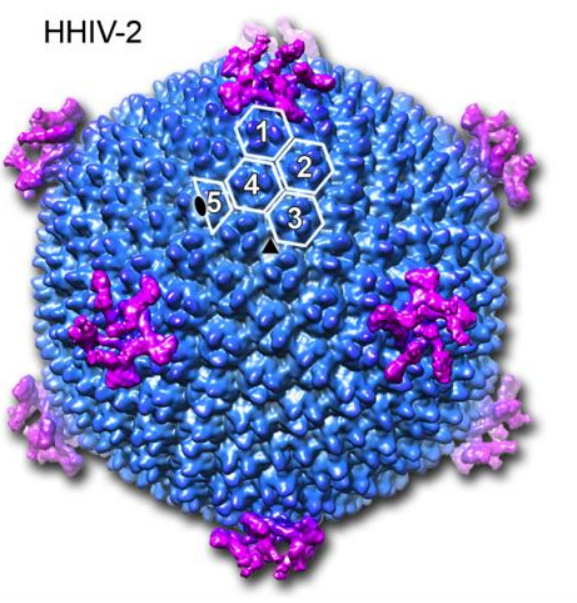

C

$\mathrm{D}$
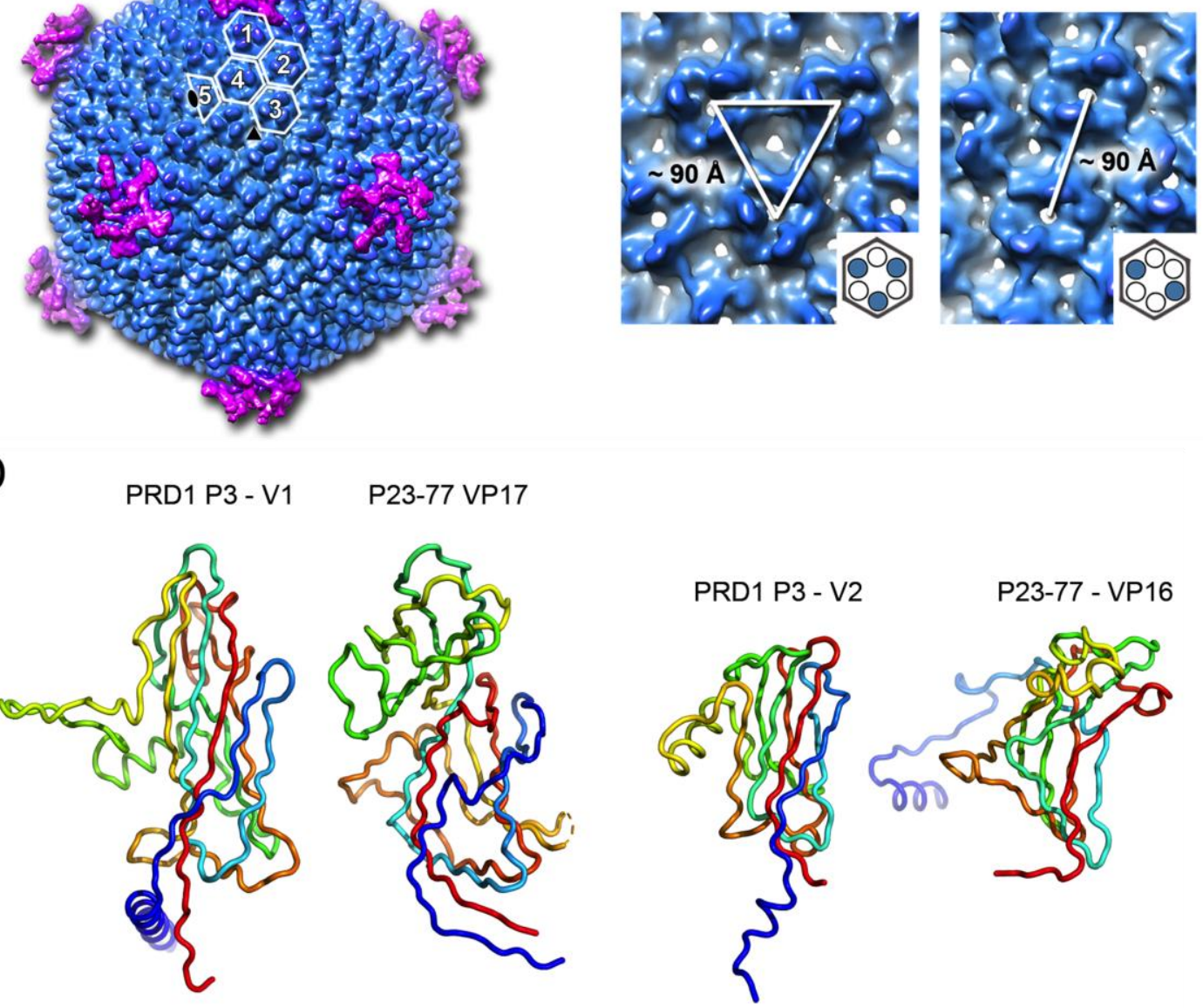

PRD1 P3 - V2

P23-77 - VP16
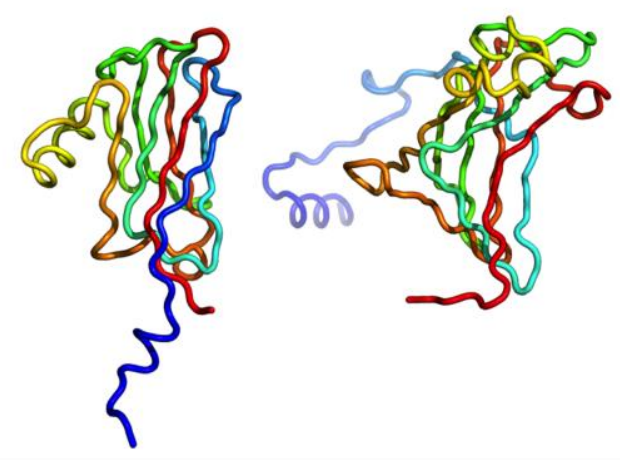

Fig. 6: Single vertical jelly roll viruses.

A) Spherolipovirus HCIV-1 genome and putative proviral genetic elements (HalaCibP1, HalaPauP1, and HaloLacP1) identified in euryarchaeal chromosomes based on sequence similarity as examples for spherolipovirus-like proviruses (Demina et al., 2017). Grey arrows indicate open reading frames 
(ORFs) or genes. Coloured arrows and arrows with colour frames indicate homologous genes known to encode structural proteins in HCIV-1 or putative homologous proteins. HCIV-1 gene numbers and structural proteins (VP), putative packaging ATPase, and the major capsid proteins (MCP) are indicated. Nucleotide coordinates are in purple. Integrase and transposase encoding genes, as well as tRNA genes, are shown in the putative proviruses. Modified from (Demina et al., 2017). Hap. cibarius, Haladaptatus cibarius; Haladaptatus paucihalophilus; Hbf. lacisalsi, Halobiforma lacisalsi. B) Overall view of cryo-EM map of vertical single $\beta$-barrel archaeal virus HHVI-2 (pseudo- $T=28$ ) at $13 \AA$ resolution (capsid, light-blue; spike proteins at the five-fold vertices, light magenta). Capsomers composing the IAU are numbered 1-5 with the two-turret capsomers labeled 4 and 5 and three-turret capsomers labeled 1-3; capsomer 5 is located on the two-fold icosahedral symmetry axis (black oval) and capsomer 3 is proximal to the three-fold axis (black triangle). C) surface rendering of the threeturret capsomers (left) and two-turret capsomers (right). The distances between the centers of the capsomers are marked (left and right panels are not to scale). Schematic diagrams (bottom right) illustrate the organization of the pseudo-hexameric capsomers (white circles, VP7; slate-blue circles, VP4). D) side-by-side comparison of individual $\beta$-barrel V1 and V2 of PRD1 MCP P3 and corresponding crystal structures of the two MCPs of Thermus phage P23-77, VP17 (PDB ID 3ZMN) and VP16 (PDB ID 3ZMO) (Rissanen et al., 2013). Atomic models are similarly oriented, displayed in cartoon tube and coloured from blue to red, from $\mathrm{N}$ - to $\mathrm{C}$-terminus.

Thermus phage P23-77 provides the first example of assembly of an icosahedral capsid lattice made of two MCP types (Rissanen et al., 2013). The capsid lattice of P23-77 consists of pseudo-hexameric capsomers with two-turret morphology (i.e. two out of six subunits have a turret on top of the lattice forming unit) (Jaatinen et al., 2008). The capsomers are formed of protein VP16 homodimers stabilized by strand swapping and VP17 monomers, both possessing a vertical single $\beta$-barrel fold (Rissanen et al., 2013). The P23-77 capsid has the same $T$ number as $\mathrm{SH} 1$ and HHIV2, pseudo $T=28$, which is unique to these viruses (Gil-Carton et al., 2015; Jaalinoja et al., 2008; Jaatinen et al., 2008), whereas Salisaeta phage SSIP-1 capsid is organized on a $T=49$ icosahedral lattice (Aalto et al., 2012). Contrary to P23-77, SH1 and HHIV-2 pseudo-hexameric capsomers display two different morphologies: capsomers either with two or three turrets, but both types are composed of MCPs VP4 and VP7 that likely adopt a vertical single $\beta$-barrel topology (Fig. 6B-C) (GilCarton et al., 2015).

The observation that P23-77 MCPs VP16 and VP17 adopt a vertical single $\beta$-barrel fold places this type of viruses as predating the advent of double $\beta$-barrel MCPs and suggests a fusion event between the MCP encoding genes (Fig. 6D) (Rissanen et al., 2013). However, while these crystal structures demonstrated the $\beta$-barrel fold, the 
principle governing the assembly of these viruses remains elusive. It has been proposed that the assembly of icosahedral membrane-containing viruses with two single vertical $\beta$-barrel MCPs may require further proteins that aid the registering of single $\beta$-barrel MCPs into pseudo-hexameric capsomers onto the membrane vesicle (Gil-Carton et al., 2015). Moreover, the pseudo-hexameric footprint of the capsomers is a unifying feature of the viruses in the PRD1-adenovirus, and is not dependent on genome type, capsid assembly mechanism, the number of MCPs, or host recognizing components. Significant efforts are underway to provide high-resolution structures by cryo-EM of whole virions which are definitely needed in order to understand how nature has solved the complexity of assembly by using two vertical single $\beta$-barrels forming pseudo-hexameric capsomers (I. Sántos-Pérez, D. Charro, D. Gil-Carton, M. Azkargorta, F. Elortza, D.H. Bamford, H.M. Oksanen, N.G. Abrescia, unpublished data).

\section{PRD1 in environmental and applied biology}

In the biosphere, phages modulate bacterial populations, which in turn influence phage distribution and abundance; in particular dsDNA phages are considered to be the most abundant viruses on Earth with dsDNA tailed phages overshadowing the environmental diversity of viruses (Chibani-Chennoufi et al., 2004) (Suttle, 2007). It has been shown that non-tailed dsDNA phages represent a profuse and ecologically important fraction of marine viral communities. Remarkably, a new virus family, termed "Autolykiviridae", was recently proposed and it contains a group of marine dsDNA viruses displaying morphological and structural features similar to PRD1 and more generally to tectiviruses (Kauffman et al., 2018). The structural homology extends beyond the double $\beta$-barrel fold of the MCP to the infection process. As in PRD1, a tail tube protrudes from one of the icosahedral vertices upon infection in order to penetrate the bacterial cell envelope. These newly discovered phages infecting potential members of Vibrionaceae, can be considered as efficient killers of marine bacteria due to their wide host spectrum, as opposed to tailed dsDNA phages with a considerably narrower host range. Autolykiviruses demonstrate that the diversity of non-tailed dsDNA viruses of prokaryotic hosts that are represented by the currently recognized families Tectiviridae, Corticoviridae, Turriviridae, and Spherolipoviridae, greatly surpasses the diversity previously observed. 
Searching for new viruses from various ecological niches has revealed previously unknown PRD1-like viruses and their new potential for applied science. New likely members of the family Tectiviridae, phage Toil and Gluconobacter phage GC1, infect oleaginous bacterium Rhodococcus opacus and Gluconobacter cerinus respectively. In the former case it has been proposed that phage Toil can be possibly used as an agent to extract biolipids from the host as starting material for biodiesel production (Gill et al., 2018). In the latter case, bacteriophage GC1 was isolated while surveying for phages infecting acetic acid bacteria which are spoilage microorganisms during wine making (Philippe et al., 2018). GC1 is the first example of a temperate tectivirus of Gram-negative bacteria and has been proposed to represent a new genus "Gammatectivirus" in the family of Tectiviridae. On the other hand, the temperate phages GIL01 and GIL16 in their lysogeny growth phase (genus Betatectivirus) have been shown to have a significant influence on the bacterial growth, sporulation rate, biofilm formation and swarming motility of Bacillus thuringiensis, all of which might be beneficial in the survival and colonization of bacteria in different environmental habitats indicating that the presence of the tectivirus might give ecological advantage for the cells (Gillis and Mahillon, 2014).

As a phage of enterobacteria, PRD1 has also been used for comparative and analytical purposes in environmental studies. It has served with single-stranded RNA bacteriophage MS2 in modelling and estimating the inactivation rates of natural sunlight and artificial light in water with the purpose of tackling other viruses and/or microorganisms (Fisher et al., 2011). Moreover, PRD1 because of its physicochemical properties and stability, is commonly used as a substitute tracer in subsurface transport and groundwater environment studies, for example to estimate expected virus removal rates occurring during on-land application of sewage during testing of aquifer recharge operations (Harvey and Ryan, 2004). Tectiviruses have shown their potential also in applied science and have been used as models for viral biosafety level 1 surrogates in water disinfection to facilitate novel strategies in providing safe water, or in Ebola outbreaks to evaluate surface disinfection efficacy, and utilized also for studies on airborne viruses (Gallandat and Lantagne, 2017; Turgeon et al., 2014). Finally, emerging interest in human gut microbiota - our internal 'biosphera' - has shown evidence of the existence of a human gut "virome" (Scarpellini et al., 2015). The composition of this virome includes also tectiviruses of which PRD1 is the representative family member. 
Overall, the above described involvement of PRD1 and more generally the presence and usage of PRD1-like viruses in multiple environmental scenarios reiterate the fundamental role that decades of genetic, biochemical and structural studies on PRD1 have also played in contributing to the understanding of current environmental models of bacteria-virus interactions.

\section{What more can we learn from PRD1?}

More than forty years have passed since PRD1 was isolated (Olsen et al., 1974). During these decades PRD1 has served as a marvellous model system for membrane-containing viruses unravelling fundamental aspects in microbiology, virology, membrane biology, structural biology and recently environmental biology. Importantly, its extensive biochemical and structural studies have led to the formulation of fundamental concepts in viral phylogeny that have revolutionised the way we 'look' at viruses and classify them. Using structure-based viral phylogeny through the systematic structural comparison of the fold of the MCPs, the vast majority of known viruses within the virophere can now be classified (Abrescia et al., 2012). This has also manifested the intricate symbiotic relationships that viruses and their corresponding hosts maintain to survive through evolutionary times.

So, is research on bacteriophage PRD1 coming to a close? Not yet. Fundamental aspects concerning the viral morphogenesis remain unknown - specifically how the membrane vesicle is formed. It is known that PRD1 selects the lipids from the host bacterial cell but the mechanisms behind this selection process and the vesicle assembly are elusive. Unravelling these mechanisms will provide insights into cellular membrane biology and vesicle biogenesis. The huge advances in electron microscopy favour this type of analysis. Particularly, cellular cryo-ET with implementation of phase-plate technology can help the dissection of this viral vesicle formation.

Moreover, while progress has been made in the understanding of the PRD1 DNA ejection, it remains unclear what triggers the opening of the end tip of the viral tube allowing the DNA to flow inside the cell and importantly whether the tail tube possesses an ordered symmetrical structure possibly similar to the complexity of the tails in tailed phages. In summary, the wealth of information accumulated on membrane-containing bacteriophage PRD1 is a valuable asset to contextualise future 
insights and illuminate on the biology of this type of viruses that have recently been shown to be abundant in seawater and in the biosphere.

\section{Conclusion}

The discovery of membrane-containing bacteriophage PRD1 has turned out to unravel important viral processes, but more significantly the way we look at and group the virosphere today. While our knowledge of the virus world remains limited, the use of structure-based virus phylogeny has already uncovered unexpected relationships across viruses infecting organisms belonging to the three domains of life. Certainly, challenges still remain in clustering for example enveloped viruses using the 'viral lineages' framework but other newly discovered virus families such as the Spherolipoviridae, displaying two vertical single $\beta$-barrel MCPs, are easily interpretable as possible predating clade of the PRD1-adeno viral lineage. The multidisciplinary research efforts and the wealth of information accumulated on PRD1 have, however, elevated this bacteriophage to a unique model system for membranecontaining dsDNA viruses, a type of virus that nowadays seems to rival at least in abundance other bacteriophages in the marine world - the place where life began.

\section{Acknowledgments}

We are grateful to Stavros Azinas and Ralf Richter for the collaborative work on virus mechanics and Dennis Bamford for insightful comments on the review. We also thank Juliet Cowper for language editing. This study was supported by the Spanish Ministerio de Economía y Competitividad (MINECO/FEDER BFU2015-64541-R), by the Basque Departamento de Desarrollo Económico e Infraestructuras (Ref: $37-$ 2017-00036) and Departamento de Educación, Política Lingüística y Cultura (Ref: PRE_2016_2_0151) to N.G.A.A. H.M.O was supported by the University of Helsinki and Academy of Finland funding for Instruct-FI research infrastructure. We thank MINECO for the Severo Ochoa Excellence Accreditation to the CIC bioGUNE (SEV2016-0644).

\section{References}

Aalto, A.P., Bitto, D., Ravantti, J.J., Bamford, D.H., Huiskonen, J.T., and Oksanen, H.M. (2012). Snapshot of virus evolution in hypersaline environments from the 
characterization of a membrane-containing Salisaeta icosahedral phage 1. Proc Natl Acad Sci U S A 109, 7079-7084.

Abad-Zapatero, C., Abdel-Meguid, S.S., Johnson, J.E., Leslie, A.G.W., Rayment, I., Rossmann, M.G., Suck, D., and Tsukihara, T. (1980). Structure of southern bean mosaic virus at 2.8 [angst] resolution. Nature 286, 33-39.

Abrescia, N.G., Bamford, D.H., Grimes, J.M., and Stuart, D.I. (2012). Structure Unifies the Viral Universe. Annu Rev Biochem 81, 795-822.

Abrescia, N.G., Cockburn, J.J., Grimes, J.M., Sutton, G.C., Diprose, J.M., Butcher, S.J., Fuller, S.D., San Martin, C., Burnett, R.M., Stuart, D.I., et al. (2004). Insights into assembly from structural analysis of bacteriophage PRD1. Nature 432, 68-74.

Abrescia, N.G., Grimes, J.M., Kivela, H.M., Assenberg, R., Sutton, G.C., Butcher, S.J., Bamford, J.K., Bamford, D.H., and Stuart, D.I. (2008). Insights into virus evolution and membrane biogenesis from the structure of the marine lipid-containing bacteriophage PM2. Mol Cell 31, 749-761.

Athappilly, F.K., Murali, R., Rux, J.J., Cai, Z., and Burnett, R.M. (1994). The refined crystal structure of hexon, the major coat protein of adenovirus type 2 , at $2.9 \mathrm{~A}$ resolution. J Mol Biol 242, 430-455.

Azinas, S., Bano, F., Torca, I., Bamford, D.H., Schwartz, G.A., Esnaola, J., Oksanen, H.M., Richter, R.P., Abrescia, N.G. (2018). Membrane-containing virus particle exhibits mechanics of a composite material for genome protection. Nanoscale 10 , 7769-7779.

Bahar, M.W., Graham, S.C., Stuart, D.I., and Grimes, J.M. (2011). Insights into the evolution of a complex virus from the crystal structure of vaccinia virus d13. Structure 19, 1011-1020.

Baker, M.L., Jiang, W., Rixon, F.J., and Chiu, W. (2005). Common ancestry of herpesviruses and tailed DNA bacteriophages. J Virol 79, 14967-14970.

Bamford, D., McGraw, T., MacKenzie, G., and Mindich, L. (1983). Identification of a protein bound to the termini of bacteriophage PRD1 DNA. J Virol 47, 311-316. 
Bamford, D., and Mindich, L. (1982). Structure of the lipid-containing bacteriophage PRD1: disruption of wild-type and nonsense mutant phage particles with guanidine hydrochloride. J Virol 44, 1031-1038.

Bamford, D.H., Burnett, R.M., and Stuart, D.I. (2002). Evolution of viral structure. Theor Popul Biol 61, 461-470.

Bamford, D.H., Ravantti, J.J., Ronnholm, G., Laurinavicius, S., Kukkaro, P., DyallSmith, M., Somerharju, P., Kalkkinen, N., and Bamford, J.K. (2005). Constituents of $\mathrm{SH} 1$, a novel lipid-containing virus infecting the halophilic euryarchaeon Haloarcula hispanica. J Virol 79, 9097-9107.

Bamford, J.K., and Bamford, D.H. (1991). Large-scale purification of membranecontaining bacteriophage PRD1 and its subviral particles. Virology 181, 348-352.

Benson, S.D., Bamford, J.K., Bamford, D.H., and Burnett, R.M. (1999). Viral evolution revealed by bacteriophage PRD1 and human adenovirus coat protein structures. Cell 98, 825-833.

Butcher, S.J., Bamford, D.H., and Fuller, S.D. (1995). DNA packaging orders the membrane of bacteriophage PRD1. Embo J 14, 6078-6086.

Caldentey, J., Blanco, L., Savilahti, H., Bamford, D.H., and Salas, M. (1992). In vitro replication of bacteriophage PRD1 DNA. Metal activation of protein-primed initiation and DNA elongation. Nucleic Acids Res 20, 3971-3976.

Cardone, G., Winkler, D.C., Trus, B.L., Cheng, N., Heuser, J.E., Newcomb, W.W., Brown, J.C., and Steven, A.C. (2007). Visualization of the herpes simplex virus portal in situ by cryo-electron tomography. Virology 361, 426-434.

Chibani-Chennoufi, S., Bruttin, A., Dillmann, M.L., and Brussow, H. (2004). Phagehost interaction: an ecological perspective. J Bacteriol 186, 3677-3686.

Cockburn, J.J., Abrescia, N.G., Grimes, J.M., Sutton, G.C., Diprose, J.M., Benevides, J.M., Thomas, G.J., Jr., Bamford, J.K., Bamford, D.H., and Stuart, D.I. (2004). Membrane structure and interactions with protein and DNA in bacteriophage PRD1. Nature 432, 122-125. 
Demina, T.A., Pietila, M.K., Svirskaite, J., Ravantti, J.J., Atanasova, N.S., Bamford, D.H., and Oksanen, H.M. (2016). Archaeal Haloarcula californiae Icosahedral Virus 1 Highlights Conserved Elements in Icosahedral Membrane-Containing DNA Viruses from Extreme Environments. MBio 7.

Demina, T.A., Pietila, M.K., Svirskaite, J., Ravantti, J.J., Atanasova, N.S., Bamford, D.H., and Oksanen, H.M. (2017). HCIV-1 and Other Tailless Icosahedral Internal Membrane-Containing Viruses of the Family Sphaerolipoviridae. Viruses 9.

Eskelin, K., Lampi, M., Meier, F., Moldenhauer, E., Bamford, D.H., and Oksanen, H.M. (2016). Asymmetric flow field flow fractionation methods for virus purification. Journal of chromatography A 1469, 108-119.

Espejo, R.T., and Canelo, E.S. (1968). Properties of bacteriophage PM2: a lipidcontaining bacterial virus. Virology 34, 738-747.

Fisher, M.B., Love, D.C., Schuech, R., and Nelson, K.L. (2011). Simulated sunlight action spectra for inactivation of MS2 and PRD1 bacteriophages in clear water. Environmental science \& technology 45, 9249-9255.

Gallandat, K., and Lantagne, D. (2017). Selection of a Biosafety Level 1 (BSL-1) surrogate to evaluate surface disinfection efficacy in Ebola outbreaks: Comparison of four bacteriophages. PLoS One 12, e0177943.

Gil-Carton, D., Jaakkola, S.T., Charro, D., Peralta, B., Castano-Diez, D., Oksanen, H.M., Bamford, D.H., and Abrescia, N.G. (2015). Insight into the Assembly of Viruses with Vertical Single beta-barrel Major Capsid Proteins. Structure 23, 1866-1877.

Gill, J.J., Wang, B., Sestak, E., Young, R., and Chu, K.H. (2018). Characterization of a Novel Tectivirus Phage Toil and Its Potential as an Agent for Biolipid Extraction. Scientific reports 8, 1062.

Gillis, A., and Mahillon, J. (2014). Prevalence, genetic diversity, and host range of tectiviruses among members of the Bacillus cereus group. Appl Environ Microbiol 80, 4138-4152.

Gowen, B., Bamford, J.K., Bamford, D.H., and Fuller, S.D. (2003). The tailless icosahedral membrane virus PRD1 localizes the proteins involved in genome packaging and injection at a unique vertex. J Virol 77, 7863-7871. 
Grahn, A.M., Daugelavicius, R., and Bamford, D.H. (2002a). Sequential model of phage PRD1 DNA delivery: active involvement of the viral membrane. Mol Microbiol 46, 1199-1209.

Grahn, A.M., Daugelavicius, R., and Bamford, D.H. (2002b). The small viral membrane-associated protein P32 is involved in bacteriophage PRD1 DNA entry. J Virol 76, 4866-4872.

Harvey, R.W., and Ryan, J.N. (2004). Use of PRD1 bacteriophage in groundwater viral transport, inactivation, and attachment studies. FEMS microbiology ecology 49 , 3-16.

Hogle, J.M., Chow, M., and Filman, D.J. (1985). Three-dimensional structure of poliovirus at 2.9 A resolution. Science 229, 1358-1365.

Hong, C., Oksanen, H.M., Liu, X., Jakana, J., Bamford, D.H., and Chiu, W. (2014). A structural model of the genome packaging process in a membrane-containing double stranded DNA virus. PLoS Biol 12, e1002024.

Huiskonen, J.T., Manole, V., and Butcher, S.J. (2007). Tale of two spikes in bacteriophage PRD1. Proc Natl Acad Sci U S A 104, 6666-6671.

Jaakkola, S.T., Penttinen, R.K., Vilen, S.T., Jalasvuori, M., Ronnholm, G., Bamford, J.K., Bamford, D.H., and Oksanen, H.M. (2012). Closely related archaeal Haloarcula hispanica icosahedral viruses HHIV-2 and SH1 have nonhomologous genes encoding host recognition functions. J Virol 86, 4734-4742.

Jaalinoja, H.T., Roine, E., Laurinmaki, P., Kivela, H.M., Bamford, D.H., and Butcher, S.J. (2008). Structure and host-cell interaction of $\mathrm{SH} 1$, a membrane-containing, halophilic euryarchaeal virus. Proc Natl Acad Sci U S A 105, 8008-8013.

Jaatinen, S.T., Happonen, L.J., Laurinmaki, P., Butcher, S.J., and Bamford, D.H. (2008). Biochemical and structural characterisation of membrane-containing icosahedral dsDNA bacteriophages infecting thermophilic Thermus thermophilus. Virology 379, 10-19.

Jaatinen, S.T., Viitanen, S.J., Bamford, D.H., and Bamford, J.K. (2004). Integral membrane protein P16 of bacteriophage PRD1 stabilizes the adsorption vertex structure. J Virol 78, 9790-9797. 
Jeembaeva, M., Jonsson, B., Castelnovo, M., and Evilevitch, A. (2010). DNA heats up: energetics of genome ejection from phage revealed by isothermal titration calorimetry. J Mol Biol 395, 1079-1087.

Jiang, W., Li, Z., Zhang, Z., Baker, M.L., Prevelige, P.E., Jr., and Chiu, W. (2003). Coat protein fold and maturation transition of bacteriophage P22 seen at subnanometer resolutions. Nat Struct Biol 10, 131-135.

Karhu, N.J., Ziedaite, G., Bamford, D.H., and Bamford, J.K. (2007). Efficient DNA packaging of bacteriophage PRD1 requires the unique vertex protein P6. J Virol 81, 2970-2979.

Kauffman, K.M., Hussain, F.A., Yang, J., Arevalo, P., Brown, J.M., Chang, W.K., VanInsberghe, D., Elsherbini, J., Sharma, R.S., Cutler, M.B., et al. (2018). A major lineage of non-tailed dsDNA viruses as unrecognized killers of marine bacteria. Nature 554, 118-122.

Khayat, R., Tang, L., Larson, E.T., Lawrence, C.M., Young, M., and Johnson, J.E. (2005). Structure of an archaeal virus capsid protein reveals a common ancestry to eukaryotic and bacterial viruses. Proc Natl Acad Sci U S A 102, 18944-18949.

Kivela, H.M., Kalkkinen, N., and Bamford, D.H. (2002). Bacteriophage PM2 has a protein capsid surrounding a spherical proteinaceous lipid core. J Virol 76, 81698178.

Kivela, H.M., Mannisto, R.H., Kalkkinen, N., and Bamford, D.H. (1999). Purification and protein composition of PM2, the first lipid-containing bacterial virus to be isolated. Virology 262, 364-374.

Laurinavicius, S., Bamford, D.H., and Somerharju, P. (2007). Transbilayer distribution of phospholipids in bacteriophage membranes. Biochim Biophys Acta 1768, 25682577.

Leigh, B., Breitbart, M.,Oksanen, H.M., Bamford, D.H. and Dishaw, L. (2018). Genome sequence of PM-like phage Cr39582 induced from Pseudoalteromonas sp. isolate from gut of Ciona robusta. Genome Announcement, in press. 
Liu, H., Jin, L., Koh, S.B., Atanasov, I., Schein, S., Wu, L., and Zhou, Z.H. (2010). Atomic structure of human adenovirus by cryo-EM reveals interactions among protein networks. Science 329, 1038-1043.

Llauro, A., Schwarz, B., Koliyatt, R., de Pablo, P.J., and Douglas, T. (2016). Tuning Viral Capsid Nanoparticle Stability with Symmetrical Morphogenesis. ACS Nano 10, 8465-8473.

Lokareddy, R.K., Sankhala, R.S., Roy, A., Afonine, P.V., Motwani, T., Teschke, C.M., Parent, K.N., and Cingolani, G. (2017). Portal protein functions akin to a DNA-sensor that couples genome-packaging to icosahedral capsid maturation. Nature communications 8, 14310 .

Mao, H., Saha, M., Reyes-Aldrete, E., Sherman, M.B., Woodson, M., Atz, R., Grimes, S., Jardine, P.J., and Morais, M.C. (2016). Structural and Molecular Basis for Coordination in a Viral DNA Packaging Motor. Cell reports 14, 2017-2029.

Martin-Gonzalez, N., Ortega-Esteban, A., Moreno-Madrid, F., Llauro, A., HernandoPerez, M., and de Pablo, P.J. (2018). Atomic Force Microscopy of Protein Shells: Virus Capsids and Beyond. Methods Mol Biol 1665, 281-296.

Mindich, L., Bamford, D., Goldthwaite, C., Laverty, M., and Mackenzie, G. (1982). Isolation of nonsense mutants of lipid-containing bacteriophage PRD1. J Virol 44, 1013-1020.

Molineux, I.J., and Panja, D. (2013). Popping the cork: mechanisms of phage genome ejection. Nat Rev Microbiol 11, 194-204.

Nandhagopal, N., Simpson, A.A., Gurnon, J.R., Yan, X., Baker, T.S., Graves, M.V., Van Etten, J.L., and Rossmann, M.G. (2002). The structure and evolution of the major capsid protein of a large, lipid-containing DNA virus. Proc Natl Acad Sci U S A 99, 14758-14763.

Newcomb, W.W., Juhas, R.M., Thomsen, D.R., Homa, F.L., Burch, A.D., Weller, S.K., and Brown, J.C. (2001). The UL6 gene product forms the portal for entry of DNA into the herpes simplex virus capsid. J Virol 75, 10923-10932.

Oksanen, H.M., Bamford D. H. (2012). Tectiviridae/PRD1 Chapter. In Virus Taxonomy : Ninth Report of the International Committee on Taxonomy of Viruses, 
M.J.A. Andrew M Q King, Eric B Carstens, Elliot J Lefkowitz, ed. (Elsevier Scientific Publ. Co), pp. 317-322.

Oksanen, H.M., Domanska, A., and Bamford, D.H. (2012). Monolithic ion exchange chromatographic methods for virus purification. Virology 434, 271-277.

Oksanen, H.M., and Ictv Report, C. (2017). ICTV Virus Taxonomy Profile: Corticoviridae. J Gen Virol 98, 888-889.

Olsen, R.H., Siak, J.S., and Gray, R.H. (1974). Characteristics of PRD1, a plasmiddependent broad host range DNA bacteriophage. J Virol 14, 689-699.

Pawlowski, A., Rissanen, I., Bamford, J.K., Krupovic, M., and Jalasvuori, M. (2014). Gammasphaerolipovirus, a newly proposed bacteriophage genus, unifies viruses of halophilic archaea and thermophilic bacteria within the novel family Sphaerolipoviridae. Arch Virol 159, 1541-1554.

Peralta, B., Gil-Carton, D., Castano-Diez, D., Bertin, A., Boulogne, C., Oksanen, H.M., Bamford, D.H., and Abrescia, N.G. (2013). Mechanism of membranous tunnelling nanotube formation in viral genome delivery. PLoS Biol 11, e1001667.

Philippe, C., Krupovic, M., Jaomanjaka, F., Claisse, O., Petrel, M., and le Marrec, C. (2018). Bacteriophage GC1, a Novel Tectivirus Infecting Gluconobacter Cerinus, an Acetic Acid Bacterium Associated with Wine-Making. Viruses 10.

Porter, K., Tang, S.-L., Chen, C.-P., Chiang, P.-W., Hong, M.-J., and Dyall-Smith, M. (2013). PH1: an archaeovirus of Haloarcula hispanica related to SH1 and HHIV-2. Archaea, 2013:456318.

Rice, G., Tang, L., Stedman, K., Roberto, F., Spuhler, J., Gillitzer, E., Johnson, J.E., Douglas, T., and Young, M. (2004). The structure of a thermophilic archaeal virus shows a double-stranded DNA viral capsid type that spans all domains of life. Proc Natl Acad Sci U S A 101, 7716-7720.

Rissanen, I., Grimes, J.M., Pawlowski, A., Mantynen, S., Harlos, K., Bamford, J.K., and Stuart, D.I. (2013). Bacteriophage P23-77 capsid protein structures reveal the archetype of an ancient branch from a major virus lineage. Structure 21, 718-726. 
Rossmann, M.G., Arnold, E., Erickson, J.W., Frankenberger, E.A., Griffith, J.P., Hecht, H.J., Johnson, J.E., Kamer, G., Luo, M., Mosser, A.G., et al. (1985). Structure of a human common cold virus and functional relationship to other picornaviruses. Nature 317, 145-153.

San Martin, C., Burnett, R. M., de Haas, F., Heinkel, R., Rutten, T., Fuller, S. D., Butcher, S. J., Bamford, D. H. (2001). Combined EM/X-Ray Imaging Yields a QuasiAtomic Model of the Adenovirus-Related Bacteriophage PRD1 and Shows Key Capsid and Membrane Interactions. Structure 9, 917-930.

San Martin, C., Huiskonen, J.T., Bamford, J.K., Butcher, S.J., Fuller, S.D., Bamford, D.H., and Burnett, R.M. (2002). Minor proteins, mobile arms and membrane-capsid interactions in the bacteriophage PRD1 capsid. Nat Struct Biol 9, 756-763.

Sántos-Pérez, I., Oksanen, H.M., Bamford, D.H., Goni, F.M., Reguera, D., and Abrescia, N.G.A. (2017). Membrane-assisted viral DNA ejection. Biochimica Et Biophysica Acta-General Subjects 1861, 664-672.

Savilahti, H., and Bamford, D.H. (1993). Protein-primed DNA replication: role of inverted terminal repeats in the Escherichia coli bacteriophage PRD1 life cycle. J Virol 67, 4696-4703.

Scarpellini, E., Ianiro, G., Attili, F., Bassanelli, C., De Santis, A., and Gasbarrini, A. (2015). The human gut microbiota and virome: Potential therapeutic implications. Digestive and liver disease : official journal of the Italian Society of Gastroenterology and the Italian Association for the Study of the Liver 47, 1007-1012.

Simpson, A.A., Tao, Y., Leiman, P.G., Badasso, M.O., He, Y., Jardine, P.J., Olson, N.H., Morais, M.C., Grimes, S., Anderson, D.L., et al. (2000). Structure of the bacteriophage phi29 DNA packaging motor. Nature 408, 745-750.

Sinclair, R.M., Ravantti, J.J., and Bamford, D.H. (2017). Nucleic and Amino Acid Sequences Support Structure-Based Viral Classification. J Virol 91.

Spencer, R. (1963). Bacterial viruses in the sea. Paper presented at: Symposium on marine microbiology (Charles $C$ Thomas). 
Stromsten, N.J., Bamford, D.H., and Bamford, J.K. (2003). The unique vertex of bacterial virus PRD1 is connected to the viral internal membrane. J Virol 77, 63146321.

Stromsten, N.J., Bamford, D.H., and Bamford, J.K. (2005). In vitro DNA packaging of PRD1: a common mechanism for internal-membrane viruses. J Mol Biol 348, 617629.

Suttle, C.A. (2007). Marine viruses--major players in the global ecosystem. Nat Rev Microbiol 5, 801-812.

Turgeon, N., Toulouse, M.J., Martel, B., Moineau, S., and Duchaine, C. (2014). Comparison of five bacteriophages as models for viral aerosol studies. Appl Environ Microbiol 80, 4242-4250.

Xu, L., Benson, S.D., Butcher, S.J., Bamford, D.H., and Burnett, R.M. (2003). The receptor binding protein $\mathrm{P} 2$ of $\mathrm{PRD} 1$, a virus targeting antibiotic-resistant bacteria, has a novel fold suggesting multiple functions. Structure 11, 309-322.

Yan, X., Olson, N.H., Van Etten, J.L., Bergoin, M., Rossmann, M.G., and Baker, T.S. (2000). Structure and assembly of large lipid-containing dsDNA viruses. Nat Struct Biol 7, 101-103.

Yan, X., Yu, Z., Zhang, P., Battisti, A.J., Holdaway, H.A., Chipman, P.R., Bajaj, C., Bergoin, M., Rossmann, M.G., and Baker, T.S. (2009). The capsid proteins of a large, icosahedral dsDNA virus. J Mol Biol 385, 1287-1299.

Zhang, X., Xiang, Y., Dunigan, D.D., Klose, T., Chipman, P.R., Van Etten, J.L., and Rossmann, M.G. (2011). Three-dimensional structure and function of the Paramecium bursaria chlorella virus capsid. Proc Natl Acad Sci U S A 108, 1483714842.

Zhang, Z., Liu, Y., Wang, S., Yang, D., Cheng, Y., Hu, J., Chen, J., Mei, Y., Shen, P., Bamford, D.H., et al. (2012). Temperate membrane-containing halophilic archaeal virus SNJ1 has a circular dsDNA genome identical to that of plasmid pHH205. Virology 434, 233-241. 
Ziedaite, G., Kivela, H.M., Bamford, J.K., and Bamford, D.H. (2009). Purified membrane-containing procapsids of bacteriophage PRD1 package the viral genome. J Mol Biol 386, 637-647. 